\title{
Motion of a sphere in the presence of a plane interface. Part 2. An exact solution in bipolar co-ordinates
}

\author{
By S. H. LEE AND L. G. LEAL \\ Department of Chemical Engineering, California Institute of Technology, \\ Pasadena, California 91125
}

(Received 22 February 1979 and in revised form 9 July 1979)

A general solution for Stokes' equation in bipolar co-ordinates is derived, and then applied to the arbitrary motion of a sphere in the presence of a plane fluid/fluid interface. The drag force and hydrodynamic torque on the sphere are then calculated for four specific motions of the sphere; namely, translation perpendicular and parallel to the interface and rotation about an axis which is perpendicular and parallel, respectively, to the interface. The most significant result of the present work is the comparison between these numerically exact solutions and the approximate solutions from part 1. The latter can be generalized to a variety of particle shapes, and it is thus important to assess their accuracy for this case of spherical particles where an exact solution can be obtained. In addition to comparisons with the approximate solutions, we also examine the predicted changes in the velocity, pressure and vorticity fields due to the presence of the plane interface. One particularly interesting feature of the solutions is the fact that the direction of rotation of a freely suspended sphere moving parallel to the interface can either be the same as for a sphere rolling along the interface (as might be intuitively expected), or opposite depending upon the location of the sphere centre and the ratio of viscosities for the two fluids.

\section{Introduction}

When a small particle is translating or rotating near a fluid/fluid interface, the hydrodynamic force and torque on the particle are changed relative to their values in an unbounded fluid. In this paper, which is the second of a three-part series, we consider the simplest problem of this type; namely, the creeping motion of a rigid spherical particle of radius $a$, whose centre is instantaneously at a distance $d(>a)$ from a flat, horizontal fluid interface. As explained in part 1 (Lee, Chadwick \& Leal 1979), this problem represents a first, asymptotic approximation which is valid when either the interfacial tension or density difference between the two fluids is large, i.e. either

$$
\frac{\mu U_{c}}{\sigma} \ll 1 \quad \text { or } \quad \frac{\mu U_{c}}{g a^{2} \Delta \rho} \ll 1,
$$

where $U_{c}$ is the appropriate characteristic velocity ( $U$ for translational motion and $\Omega a$ for rotational motion) and the remaining variables are as defined in part 1 . In any of these circumstances, a real fluid interface will remain only slightly deformed and in quasi-static equilibrium with the flow-induced stress fields in the two fluids. 0022-1120/80/4406-8570\$02.00 (C) 1980 Cambridge University Press 
In part 1 we obtained approximate solutions for the same problem when the sphere is far from the interface,

$$
l \equiv d / a \gg 1,
$$

using a generalization of the method of Lorentz (1907). Although the condition (2) is independently sufficient for small interface deformation, the solutions in part 1 still require the conditions (1) to be satisfied since the interface was assumed to be flat at all orders of approximation in $l^{-1}$. In the present paper, we obtain exact solutions for the motion of a sphere near a flat fluid interface using a series expansion of eigensolutions in bipolar (spherical) co-ordinates. These exact solutions extend the domain of allowable $l$ relative to the condition (2) and thus serve to clarify some physical features of the motion of spherical particles near a fluid interface. More importantly, however, these exact solutions provide a basis for evaluating the accuracy of the asymptotic solutions as a function of $l$. We have noted in part 1 that the approximate solution technique can be applied in a straightforward fashion to other particle shapes unlike the exact eigensolution expansion of the present paper which is strictly limited to spherical particles. Such generalizations could be of considerable significance in a number of applications, but only if the approximate solutions exhibit reasonable accuracy for most of the range of possible values of $l$ - certainly a big 'if' in view of the condition (2). Comparison of the exact solutions obtained here with the approximate solutions of part 1 is particularly significant since the case of a spherical particle is the only one where exact solutions are possible for arbitrary particle motions and arbitrary values of the viscosity ratio, $\lambda$.

The use of bipolar (spherical) co-ordinates in low-Reynolds-number hydrodynamics was initiated by Jeffery $(1912,1915)$ who first derived eigensolutions of Laplace's equation and then used these eigensolutions to analyse the fluid motion generated by two spheres which rotate about their line of centres. Later, Stimson \& Jeffery (1926) used a stream function expansion in bipolar co-ordinates to solve the problem of two spheres translating along their line of centres with the same constant velocity. This work was extended by Bart (1968) to calculate the drag force on a spherical drop which is translating normal to a flat fluid/fluid interface. A more difficult extension to the non-axisymmetric problem of a sphere rotating or translating parallel to a plane solid wall was accomplished by Dean \& O'Neill (1963) and O'Neill (1964), respectively.

In this paper, we generalize the solutions of Jeffery (1912) and Dean \& O'Neill (1963) to consider arbitrary translational or rotational motions of a rigid sphere in the presence of a plane fluid/fluid interface. We begin, in $\S 2.1$ by deriving a general, infinite series solution for Stokes' equation in terms of eigensolutions for bipolar (spherical) co-ordinates. When this solution is applied to the present class of problems, it is shown in $\$ 2.2$ that a complete numerical specification of the velocity and pressure fields requires the solution of an infinite set of algebraic equations for the coefficients of this series. Fortunately, in general, the magnitude of the various terms in the infinite series decreases exponentially with increasing order, and any desired degree of numerical accuracy can thus be achieved by retaining only a finite number of terms. Since the rate of convergence does decrease as $l$ decreases, it is necessary to retain a larger number of terms to yield the same numerical accuracy in the results as the sphere moves closer to the interface. This is not a serious limitation, however, 
since the numerical evaluation of coefficients in the truncated series reduces to the solutions of a band matrix and there is relatively little cost in computation time to include a large number of terms. After generating the general solution, as described above, it is applied in $\S 4.1$ to the four fundamental problems of particle translation and rotation perpendicular and parallel to the fluid interface. As discussed in part 1, the linearity of the Stokes' equations and boundary conditions allows any arbitrary motion to be described in terms of a set of three hydrodynamic resistance tensors, and these tensors can be specified completely from the solutions for the four fundamental particle motions. The only significant deviation from the solution scheme described above occurs for rotation of the sphere with the axis of rotation normal to the fluid interface. In this case, there is only one non-zero velocity component, $v_{\phi}$, and the coefficients of the solution can be obtained analytically. The axisymmetric problem of translation normal to the interface can, of course, also be solved using a stream function as in Bart (1968). This alternative scheme for axisymmetric flows is discussed briefly in $\S 3$, and is shown to give identical results for axisymmetric flows to the general solution derived here. It may be noted that Bart's solution for translation normal to the interface is incomplete since it is limited to the drag force on the sphere, without explicit determination of the velocity and pressure fields. Finally, the general formulae for the force and torque on the sphere are evaluated numerically in $\S 4.2$, for viscosity ratios $\lambda=0,0 \cdot 1,1,10$ and $\infty$ and $1 \cdot 1 \leqslant l \leqslant 10$, and compared with the approximate results of part 1 . Certain general features of the solutions, notably the rotation direction for a freely suspended sphere in parallel translation, are also discussed in $\S 4.2$.

\section{Governing equations - the method of solution}

\subsection{A general solution of Stokes' equation in bipolar co-ordinates}

We begin by deriving a general solution of Stokes' equation, plus the continuity equation

$$
\begin{aligned}
& \nabla^{2} \mathbf{u}=\nabla \boldsymbol{p}, \\
& \nabla \cdot \mathbf{u}=\mathbf{0},
\end{aligned}
$$

in terms of the fundamental eigensolutions for bipolar co-ordinates. For convenience, all variables are considered to be non-dimensionalized with respect to arbitrary characteristic variables; $L_{c}, U_{c}$ and $p_{c}$ ( $\equiv \mu U_{c} / L_{c}$ ). A deseription of the bipolar coordinate system $(\xi, \eta, \phi)$ is given by Happel \& Brenner (1973). In the application of our general solution to the motion of a spherical particle near a plane interface, we shall identify the interface with the co-ordinate surface $\eta=0$ and the sphere with the co-ordinate surface $\eta=\eta_{0}=-\cosh ^{-1}(l)$. Although we could solve for the velocity components in this bipolar system directly, it is more convenient for our purposes to use the bipolar eigensolutions to evaluate the velocity components in the related cylindrical co-ordinates, $(r, z, \phi)$, which are sketched together with $(\xi, \eta, \phi)$ in figure 1 . The bipolar and cylindrical co-ordinates are related via the transformation laws

$$
z=c \frac{\sinh \eta}{\cosh \eta-\cos \xi} \text { and } r=c \frac{\sin \xi}{\cosh \eta-\cos \xi}
$$




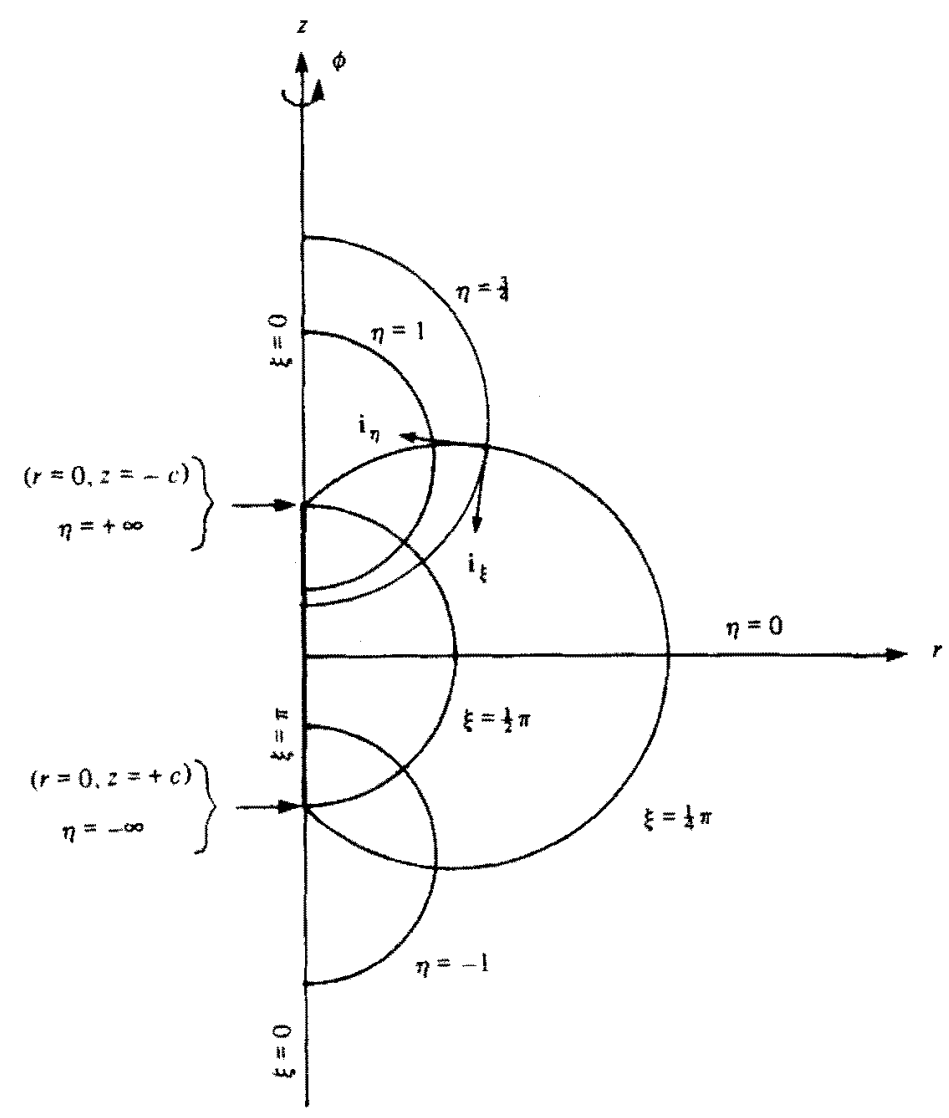

Fraure 1. Bipolar co-ordinates $(\eta, \xi, \phi)$.

in which $c$ is a constant which can be determined by the relative location of the boundaries $\eta=0$ and $\eta=\eta_{0}$ (see $\S 4$ ).

It is convenient to consider the velocity field $\mathbf{u}$ as the sum of a homogeneous and particular solution of (3) and (4). In order to determine $\mathbf{u}$, we thus require a general expression for the pressure field $p$. According to (3) and (4), $p$ is a harmonic function, i.e.

$$
\nabla^{2} p=0
$$

and can therefore be expressed in terms of Jeffery's (1912) general solution of Laplace's equation in bipolar co-ordinates

in which

$$
p=\sum_{m=0}^{\infty} p_{m}(\eta, \xi) \cos \left(m \phi+\alpha_{m}\right)
$$

$p_{m}(\eta, \xi)=(\cosh \eta-\zeta)^{\frac{1}{2}} \sum_{m=0}^{\infty}\left[A_{n}^{m} \sinh \left(n+\frac{1}{2}\right) \eta+B_{n}^{m} \cosh \left(n+\frac{1}{2}\right) \eta\right]\left[a_{n}^{m} P_{n}^{m}(\zeta)+b_{n}^{m} Q_{n}^{m}(\zeta)\right]$.

Here, $P_{n}^{m}(\zeta)$ and $Q_{n}^{m}(\zeta)$ are associated Legendre functions of the first and second kind, with argument $\zeta \equiv \cos \xi$. Since $Q_{n}^{m}(\zeta)$ increases to infinity on the $z$ axis, we require

$$
b_{n}^{m} \equiv 0
$$


in $p_{m}(\eta, \xi)$, thus yielding

$$
p_{m}(\eta, \xi)=\frac{1}{c}(\cosh \eta-\zeta)^{\frac{1}{2}} \sum_{n=m}^{\infty}\left[A_{n}^{m} \sinh \left(n+\frac{1}{2}\right) \eta+B_{n}^{m} \cosh \left(n+\frac{1}{2}\right) \eta\right] P_{n}^{m}(\zeta)
$$

Let us now consider the solution of (3) and (4) with $p$ given by (7) and (8). With the components of $\mathbf{u}$ in the $(r, \phi, z)$ directions denoted, respectively, as $u, v$ and $w$, the equations (3) and (4) can be written as

$$
\begin{aligned}
\frac{\partial p}{\partial r} & =\left(\nabla^{2}-\frac{1}{r^{2}}\right) u-\frac{2}{r^{2}} \frac{\partial v}{\partial \phi} \\
\frac{1}{r} \frac{\partial p}{\partial \phi} & =\left(\nabla^{2}-\frac{1}{r^{2}}\right) v+\frac{2}{r^{2}} \frac{\partial u}{\partial \phi} \\
\frac{\partial p}{\partial z} & =\nabla^{2} w
\end{aligned}
$$

and

with

$$
\frac{1}{r} \frac{\partial}{\partial r}(r u)+\frac{1}{r} \frac{\partial v}{\partial \phi}+\frac{\partial w}{\partial z}=0
$$

$$
\nabla^{2} \equiv\left(\frac{\partial^{2}}{\partial r^{2}}+\frac{1}{r} \frac{\partial}{\partial r}+\frac{1}{r^{2}} \frac{\partial^{2}}{\partial \phi^{2}}+\frac{\partial^{2}}{\partial z^{2}}\right)
$$

Now, a particular solution of (9)-(11) is simply

where $\mathbf{x}=\left(\boldsymbol{r} \mathbf{i}_{r}+z \mathbf{i}_{z}\right)$.

$$
\mathbf{u}^{p}=\frac{1}{2} p \mathbf{x}
$$

Thus, it is necessary to solve only the homogeneous equations, (9)-(11),

$$
\begin{aligned}
& 0=\left(\nabla^{2}-\frac{1}{r^{2}}\right) u^{h}-\frac{2}{r^{2}} \frac{\partial v^{h}}{\partial \phi}, \\
& 0=\left(\nabla^{2}-\frac{1}{r^{2}}\right) v^{h}+\frac{2}{r^{2}} \frac{\partial u^{h}}{\partial \phi}, \\
& 0=\nabla^{2} w^{h},
\end{aligned}
$$

subject to (12).

The solution for $w^{h}$, which is bounded on the $z$ axis, can be readily obtained from the solution for $p$.

$$
\begin{aligned}
& w^{h}= \sum_{m} w_{m}(\eta, \xi) \cos \left(m \phi+\alpha_{m}\right) \\
&=(\cosh \eta-\zeta)^{\frac{1}{2}} \sum_{m=0}^{\infty} \sum_{n=m}^{\infty}\left[C_{n}^{m} \sinh \left(n+\frac{1}{2}\right) \eta+D_{n}^{m} \cosh \left(n+\frac{1}{2}\right) \eta\right] \\
& \quad \times P_{n}^{m}(\zeta) \cos \left(m \phi+\alpha_{m}\right) .
\end{aligned}
$$

To obtain corresponding solutions for $u^{h}$ and $v^{h}$, let us introduce the series expansion

$$
\begin{aligned}
u^{h} & =\sum_{m} u_{m}(\eta, \xi) \cos \left(m \phi+\alpha_{m}\right), \\
v^{h} & =\sum_{m} v_{m}(\eta, \xi) \sin \left(m \phi+\alpha_{m}\right) .
\end{aligned}
$$


Substituting (18) and (19) into the equations (14) and (15), we obtain

$$
\begin{aligned}
& {\left[\left(\frac{\partial^{2}}{\partial r^{2}}+\frac{1}{r} \frac{\partial}{\partial r}+\frac{\partial^{2}}{\partial z^{2}}\right)-\frac{m^{2}+1}{r^{2}}\right] u_{n m}-\frac{2}{r^{2}} m v_{m}=0} \\
& {\left[\left(\frac{\partial^{2}}{\partial r^{2}}+\frac{1}{r} \frac{\partial}{\partial r}+\frac{\partial^{2}}{\partial z^{2}}\right)-\frac{m^{2}+1}{r^{2}}\right] v_{m}-\frac{2}{r^{2}} m u_{m}=0}
\end{aligned}
$$

When $m=0$, these equations are easily solved using Jeffery's (1912) solution,

$$
\begin{aligned}
& u_{0}=(\cosh \eta-\zeta)^{\frac{1}{2}} \sum_{n=1}^{\infty}\left[E_{n}^{0} \sinh \left(n+\frac{1}{2}\right) \eta+F_{n}^{0} \cosh \left(n+\frac{1}{2}\right) n\right] P_{n}^{1}(\zeta), \\
& v_{0}=(\cosh \eta-\zeta)^{\frac{1}{2}} \sum_{n=1}^{\infty}\left[G_{n}^{0} \sinh \left(n+\frac{1}{2}\right) \eta+H_{n}^{0} \cosh \left(n+\frac{1}{2}\right) \eta\right] P_{n}^{1}(\zeta) .
\end{aligned}
$$

For $m \geqslant 1$, the addition and subtraction of equations (20) and (21) yields (cf. Dean \& O'Neill 1963; Lin 1968)

$$
\begin{aligned}
& {\left[\frac{\partial^{2}}{\partial r^{2}}+\frac{1}{r} \frac{\partial}{\partial r}+\frac{\partial^{2}}{\partial z^{2}}-\frac{1}{r^{2}}(m+1)^{2}\right] \gamma_{m}=0,} \\
& {\left[\frac{\partial^{2}}{\partial r^{2}}+\frac{1}{r} \frac{\partial}{\partial r}+\frac{\partial^{2}}{\partial z^{2}}-\frac{1}{r^{2}}(m-1)^{2}\right] \chi_{m}=0,}
\end{aligned}
$$

where $\gamma_{m} \equiv u_{m}+v_{m}$ and $\chi_{m} \equiv u_{m}-v_{m}$. The solutions of equations (24) and (25) can again be seen from Jeffery's (1912) results to be

$$
\begin{aligned}
& \gamma_{m}=(\cosh \eta-\zeta)^{\frac{1}{2}} \sum_{n=m+1}^{\infty}\left[E_{n}^{m} \sinh \left(n+\frac{1}{2}\right) \eta+F_{n}^{m} \cosh \left(n+\frac{1}{2}\right) \eta\right] P_{n}^{m+1}(\zeta), \\
& \chi_{m}=(\cosh \eta-\zeta)^{\frac{1}{2}} \sum_{n=m-1}^{\infty}\left[G_{n}^{m} \sinh \left(n+\frac{1}{2}\right) \eta+H_{n}^{m} \cosh \left(n+\frac{1}{2}\right) \eta\right] P_{n}^{m-1}(\zeta) .
\end{aligned}
$$

Hence, a general solution of Stokes' equation for the velocity components $(u, v, w)$ of the circular cylindrical co-ordinate system shown in figure 1 is simply

$$
\begin{aligned}
& u=\frac{r p}{2}+u_{0} \cos \alpha_{0}+\frac{1}{2} \sum_{m=1}^{\infty}\left(\gamma_{m}+\chi_{m}\right) \cos \left(m \phi+\alpha_{m}\right), \\
& v=v_{0} \sin \alpha_{0}+\frac{1}{2} \sum_{m=1}^{\infty}\left(\gamma_{m}-\chi_{m}\right) \sin \left(m \phi+\alpha_{m}\right), \\
& w=\frac{z p}{2}+\sum_{m=0}^{\infty} w_{m} \cos \left(m \phi+\alpha_{m}\right),
\end{aligned}
$$

in which $p, u_{0}, v_{0}, w_{m}, \gamma_{m}$ and $\chi_{m}$ are conveniently expressed for present purposes in terms of the eigensolutions for bipolar co-ordinates which are defined in terms of the cylindrical co-ordinates by the equations (5). Furthermore, the velocity components $(u, v, w)$ should satisfy the equation of continuity. Thus, substituting the equations (28), (29) and (30) into equation (12), this condition can be expressed in the form

and

$$
\left(3+r \frac{\partial}{\partial r}+z \frac{\partial}{\partial z}\right) p_{0}+2\left(\frac{\partial}{\partial r}+\frac{1}{r}\right) u_{0}+2 \frac{\partial w_{0}}{\partial z}=0 \quad \text { for } \quad m=0
$$

$\left(3+r \frac{\partial}{\partial r}+z \frac{\partial}{\partial z}\right) p_{m}+\left(\frac{\partial}{\partial r}+\frac{m+1}{r}\right) \gamma_{m}+\left(\frac{\partial}{\partial r}-\frac{m-1}{r}\right) \chi_{m}+2 \frac{\partial w_{m}}{\partial z}=0 \quad$ for $\quad m \geqslant 1$ 
Hence, we have derived a general solution of Stokes' equation, (28), (29) and (30), which is subject to the condition of mass continuity, (31 $a)$ and $(31 b)$.

\subsection{Application of the general solution to the motion of a sphere near a plane interface}

In this section, we apply the general, bipolar co-ordinate solution which was derived in the last section to determine the velocity and pressure fields for an arbitrary translational or rotational motion of a sphere near a plane fluid/fluid interface. The characteristic scales, which are inherent in (3), (4) and the subsequent solution, can be defined in this case as $L_{c}=a$ (sphere radius), $U_{c}=U$ (translational velocity of the sphere) or $\Omega a$ ( $\Omega=$ angular velocity of the sphere) and $p_{c}=\mu U_{c} / L_{c}$ or $\hat{\mu} U_{c} / L_{c}(\mu$ is the viscosity of the lower fluid and $\hat{\mu}$ is that of the upper fluid). In order to complete the specification of the problem, we consider that the sphere is located in the lower fluid and denote the viscosity ratio between the upper and lower fluids as $\lambda$.

The general solution of the preceding section, which applies separately in the upper and lower fluids, contains eight unknown coefficients, namely $A_{n}^{m}, B_{n}^{m}, \ldots, H_{n}^{m}$, for each set of $n$ and $m$, as may be seen from equations (8), (17), (22), (23), (26) and (27). Thus, including the solution constants for both fluids, we have 16 sets of unknowns to be determined in order to completely specify the velocity and pressure fields. Hereafter, the constants in the lower fluid will be denoted as $A_{n}^{m}, B_{n}^{m}, \ldots, H_{n}^{m}$, while those for the upper fluid are $\hat{A}_{n}^{m}, \hat{B}_{n}^{m}, \ldots, \hat{H}_{n}^{m}$. Two types of conditions remain to determine these constants. First are the boundary conditions at the sphere surface and the interface, and second, the general conditions of mass continuity, equation $(31 a, b)$, and boundedness of the velocities and pressure, which apply independent of the details of the boundary conditions. We may begin by examining the last two conditions first.

Let us start with the conditions which are required of the unknown constants to insure that the velocity and pressure fields are everywhere bounded. The necessity for such a condition arises from the fact that $\eta \rightarrow \pm \infty$ as $z \rightarrow \pm c$ on the $z$ axis. In the lower fluid, this causes no difficulty since the point $z=-c, r=0$ lies inside the particle. In the upper fluid, however, we must require

$$
\hat{A}_{n}^{m}=-\hat{B}_{n}^{m}, \quad \hat{C}_{n}^{m}=-\hat{D}_{n}^{m}, \quad \hat{E}_{n}^{m}=-\hat{F}_{n}^{m} \quad \text { and } \quad \hat{G}_{n}^{m}=-\hat{H}_{n}^{m}
$$

for all $n, m$ in order to insure that the velocities remain bounded as $z \rightarrow c$ on the $z$ axis.

The second general condition on the solution constants arises from the continuity equation $(31 a, b)$ which must be satisfied in both fluids. Thus, substituting equations $(8),(17),(22),(23),(26)$ and (27) into (31 $a)$ and (31b), we obtain two algebraic relationships among the constants which must be satisfied for arbitrary $\eta$ and $\xi$ (cf. Dean \& O'Neill 1963; Lin 1968). For $m=0$, we obtain for the lower fluid

$$
\begin{aligned}
-\frac{1}{2} n A_{n-1}^{0} & +\frac{5}{2} A_{n}^{0}+\frac{1}{2}(n+1) A_{n+1}^{0}-n D_{n-1}^{0}+(2 n+1) D_{n}^{0}-(n+1) D_{n+1}^{0} \\
& +n(n-1) E_{n-1}^{0}-2 n(n+1) E_{n}^{0}+(n+1)(n+2) E_{n+1}^{0}=0 \\
-\frac{1}{2} n B_{n-1}^{0} & +\frac{5}{2} B_{n}^{0}+\frac{1}{2}(n+1) B_{n+1}^{0}-n C_{n-1}^{0}+(2 n+1) C_{n}^{0}-(n+1) C_{n+1}^{0} \\
& +n(n-1) F_{n-1}^{0}-2 n(n+1) F_{n}^{0}+(n+1)(n+2) F_{n+1}^{0}=0
\end{aligned}
$$


while for $m \geqslant 1$, we find

$$
\begin{gathered}
-\frac{1}{2}(n-m) A_{n-1}^{m}+\frac{5}{2} A_{n}^{m}+\frac{1}{2}(n+m+1) A_{n+1}^{m}-(n-m) D_{n-1}^{m}+(2 n+1) D_{n}^{m} \\
-(n+m+1) D_{n+1}^{m}+\frac{1}{2}(n-m)(n-m-1) E_{n-1}^{m}-(n-m)(n+m+1) E_{n}^{m} \\
+\frac{1}{2}(n+m+1)(n+m+2) E_{n+1}^{m}-\frac{1}{2} G_{n-1}^{m}+G_{n}^{m}-\frac{1}{2} G_{n+1}^{m}=0 \\
-\frac{1}{2}(n-m) B_{n-1}^{m}+\frac{5}{2} B_{n}^{m}+\frac{1}{2}(n+m+1) B_{n+1}^{m}-(n-m) C_{n-1}^{m}+(2 n+1) C_{n}^{m} \\
-(n+m+1) C_{n+1}^{m}+\frac{1}{2}(n-m)(n-m-1) F_{n-1}^{m}-(n-m)(n+m+1) F_{n}^{m} \\
+\frac{1}{2}(n+m+1)(n+m+2) F_{n+1}^{m}-\frac{1}{2} H_{n-1}^{m}+H_{n}^{m}-\frac{1}{2} H_{n+1}^{m}=0 .
\end{gathered}
$$

Similarly, in the upper fluid, we obtain

$$
\begin{aligned}
& -\frac{1}{2} n \hat{A}_{n-1}^{0}+\frac{5}{2} \hat{A}_{n}^{0}+\frac{1}{2}(n+1) \hat{A}_{n+1}^{0}-n \hat{D}_{n-1}^{0}+(2 n+1) \hat{D}_{n}^{0} \\
& -(n+1) \hat{D}_{n+1}^{0}+n(n-1) \hat{E}_{n-1}^{0}-2 n(n+1) \hat{E}_{n}^{0}+(n+1)(n+2) \widehat{E}_{n+1}^{0}=0 \quad \text { for } m=0,
\end{aligned}
$$

and

$$
\begin{aligned}
& -\frac{1}{2}(n-m) \hat{A}_{n-1}^{m}+\frac{5}{2} \hat{A}_{n}^{m}+\frac{1}{2}(n+m+1) \hat{A}_{n+1}^{m}-(n-m) \hat{D}_{n-1}^{m}+(2 n+1) \hat{D}_{n}^{m} \\
& \quad-(n+m+1) \hat{D}_{n+1}^{m}+\frac{1}{2}(n-m)(n-m-1) \hat{E}_{n-1}^{m}-(n-m)(n+m+1) \hat{E}_{n}^{m} \\
& \quad+\frac{1}{2}(n+m+1)(n+m+2) \hat{E}_{n+1}^{m}-\frac{1}{2} \widehat{G}_{n-1}^{m}+\hat{G}_{n}^{m}-\frac{1}{2} \hat{G}_{n+1}^{m}=0 \text { for } m \geqslant 1 .
\end{aligned}
$$

It may be noted that the two equations, corresponding to $(34 a)$ and $(34 b)$ for the lower fluid, are not listed since they are not independent of $(35 a)$ and $(35 b)$ due to the conditions (32).

The conditions (32) and (33)-(35) apply for any regular motion of an incompressible fluid. The remaining conditions, however, depend upon the boundary conditions at the sphere surface and at the interface. Let us first consider the boundary conditions at the interface. For a plane interface, these conditions are zero normal velocity

$$
w=\hat{w}=0 \quad \text { on } \quad \eta=0
$$

plus continuity of the tangential components of velocity and stress. Continuity of normal stress does not enter explicitly owing to the assumption of a flat interface at this level of approximation, but would yield the first correction to the assumed interface shape owing to the motion of the two contiguous fluids. $\uparrow$ The condition (36) is satisfied trivially if

$$
D_{n}^{m}=\hat{D}_{n}^{m}=0 \text { for all } n, m .
$$

The conditions for continuity of the tangential velocity components

$$
u=\hat{u}, \quad \text { and } \quad v=\hat{v} \quad \text { on } \quad \eta=0
$$

can be reduced by substituting (28) and (29) into (38). This yields

$$
\begin{aligned}
& u_{0}-\hat{u}_{0}=-\frac{r}{2}\left(p_{0}-\hat{p}_{0}\right) \quad \text { and } \quad v_{0}=\hat{v}_{0} \text { for } m=0, \\
& \gamma_{m}-\hat{\gamma}_{m}=\chi_{m}-\hat{\chi}_{m}=-\frac{r}{2}\left(p_{m}-\hat{p}_{m}\right) \text { for } m \geqslant 1 .
\end{aligned}
$$

$\dagger$ Cf. $\$ 2$ of part 1 , where conditions are given which must be satisfied in order that the flat interface problem be a valid first approximation to the exact problem in which the interface is deformed. 
To satisfy equations $(39 a)$ and $(39 b)$ for all $\eta$ and $\xi$, we require:

$$
\begin{aligned}
& -\frac{(n-m-1)}{2 n-1}\left(F_{n-1}^{m}-\hat{F}_{n-1}^{m}\right)+\left(F_{n}^{m}-\hat{F}_{n}^{m}\right)-\frac{n+m+2}{2 n+3}\left(F_{n+1}^{m}-\hat{F}_{n+1}^{m}\right) \\
& =-\frac{1}{2}\left[\frac{1}{2 n-1}\left(B_{n-1}^{m}-\hat{B}_{n-1}^{m}\right)-\frac{1}{2 n+3}\left(B_{n+1}^{m}-\hat{B}_{n+1}^{m}\right)\right] \text { for all } m \\
& H_{n}^{0}=\hat{H}_{n}^{0} \quad \text { for } m=0 \\
& -\frac{(n-m+1)}{2 n-1}\left(H_{n-1}^{m}-\hat{H}_{n-1}^{m}\right)+\left(H_{n}^{m}-\hat{H}_{n}^{m}\right)-\frac{n+m}{2 n+3}\left(H_{n+1}^{m}-\hat{H}_{n+1}^{m}\right) \\
& =-\frac{1}{2}\left[-\frac{(n-m)(n-m+1)}{2 n-1}\left(B_{n-1}^{m}-\hat{B}_{n-1}^{m}\right)\right. \\
& \left.\quad+\frac{(n+m)(n+m+1)}{2 n+3}\left(B_{n+1}^{m}-\hat{B}_{n+1}^{m}\right)\right] \text { for } m \geqslant 1
\end{aligned}
$$

Finally, the condition of continuity of shear stress at the interface can be expressed in the form

$$
\frac{\partial u}{\partial z}=\lambda \frac{\partial \hat{u}}{\partial z} \quad \text { and } \quad \frac{\partial v}{\partial z}=\lambda \frac{\partial \hat{v}}{\partial z} \quad \text { on } \quad \eta=0
$$

Hence, using the general expressions for $u, \hat{u}, v$ and $\hat{v}$, equation (42) becomes

$$
\begin{aligned}
& \left.\begin{array}{l}
\frac{\partial}{\partial z}\left(u_{0}-\lambda \hat{u}_{0}\right)=-\frac{r}{2} \frac{\partial}{\partial z}\left(p_{0}-\lambda \hat{p}_{0}\right) \\
\frac{\partial v_{0}}{\partial z}=-\lambda \frac{\partial \hat{v}_{0}}{\partial z}
\end{array}\right\} \text { for } m=0 \\
& \frac{\partial}{\partial z}\left(\gamma_{m}-\lambda \hat{\gamma}_{m}\right)=\frac{\partial}{\partial z}\left(\chi_{m}-\lambda \hat{\chi}_{m}\right)=-\frac{r}{2} \frac{\partial}{\partial z}\left(p_{m}-\lambda \hat{p}_{m}\right) \text { for } m \geqslant 1 .
\end{aligned}
$$

From equations $(43 a)$ and $(43 b)$, we can readily derive the following additional relationships among coefficients:

$$
\begin{gathered}
-(n-m-1)\left(E_{n-1}^{m}-\lambda \widehat{E}_{n-1}^{m}\right)+(2 n+1)\left(E_{n}^{m}-\lambda \widehat{E}_{n}^{m}\right)-(n+m+2)\left(E_{n+1}^{m}-\lambda \hat{E}_{n+1}^{m}\right) \\
=-\left(A_{n-1}^{m}-\lambda \hat{A}_{n-1}^{m}\right)+\left(A_{n+1}^{m}-\lambda \hat{A}_{n+1}^{m}\right) \text { for all } m ; \\
G_{n}^{0}=\lambda \widehat{G}_{n}^{0} \text { for } m=0, \\
-(n-m+1)\left(G_{n-1}^{m}-\lambda \hat{G}_{n-1}^{m}\right)+(2 n+1)\left(G_{n}^{m}-\lambda \hat{G}_{n}^{m}\right)-(n+m)\left(G_{n+1}^{m}-\lambda \hat{G}_{n+1}^{m}\right) \\
=(n-m)(n-m+1)\left(A_{n-1}^{m}-\lambda \hat{A}_{n-1}^{m}\right)-(n+m)(n+m+1)\left(A_{n+1}^{m}-\lambda \hat{A}_{n+1}^{m}\right) \\
\text { for } m \geqslant 1 .
\end{gathered}
$$

The final step in obtaining a solution is to apply the 'no-slip' and kinematic boundary conditions at the sphere surface, i.e.

$$
\mathbf{u}=\mathbf{u}_{s} \text { at } \eta=\eta_{0} .
$$

The most convenient method for doing this is to express $\mathbf{u}_{s}$ in terms of the bipolar eigenfunctions. Thus, we first expand the three components of $\mathbf{u}_{s}$ as

$$
u_{s}=\sum_{m} u_{s}^{m}(\eta, \xi) \cos \left(m \phi+\alpha_{m}\right)
$$




$$
\begin{aligned}
v_{s} & =\sum_{m} v_{s}^{m}(\eta, \xi) \sin \left(m \phi+\alpha_{m}\right), \\
w_{s} & =\sum_{m} w_{s}^{m}(\eta, \xi) \cos \left(m \phi+\alpha_{m}\right) .
\end{aligned}
$$

Then, for $m=0$, we may further expand the functions $u_{s}^{0}$ and $v_{s}^{0}$ as

while for $m \geqslant 1$,

$$
\begin{aligned}
& u_{s}^{0}=\left(\cosh \eta_{0}-\zeta\right)^{\frac{1}{2}} \Sigma X_{n}^{0}(\eta) P_{n}^{1}(\zeta), \\
& v_{s}^{0}=\left(\cosh \eta_{0}-\zeta\right)^{\frac{1}{2}} \Sigma Y_{n}^{0}(\eta) P_{n}^{1}(\zeta) ;
\end{aligned}
$$

In addition, for all $m$,

$$
\begin{aligned}
& u_{s}^{m}+v_{s}^{m}=\left(\cosh \eta_{0}-\zeta\right)^{\frac{1}{2}} \Sigma X_{n}^{m}(\eta) P_{n}^{m+1}(\zeta) \\
& u_{s}^{m}-v_{s}^{m}=\left(\cosh \eta_{0}-\zeta\right)^{\frac{1}{2}} \Sigma Y_{n}^{m}(\eta) P_{n}^{m-1}(\zeta)
\end{aligned}
$$

$$
w_{n}^{m}=\left(\cosh \eta_{0}-\zeta\right)^{\frac{1}{2}} \Sigma Z_{n}^{m}(\eta) P_{n}^{m}(\zeta)
$$

Now, using the general solution for the velocity components, $(28)-(30)$, and the condition (46), we obtain, for $m=0$,

and for $m \geqslant 1$,

$$
\begin{aligned}
& u_{0}=u_{s}^{0}-\frac{r}{z}\left(w_{s}^{0}-w_{0}\right), \\
& v_{0}=v_{s}^{0}
\end{aligned}
$$

$$
\begin{aligned}
& \gamma_{m}=u_{s}^{m}+v_{s}^{m}-\frac{r}{z}\left(w_{s}^{m}-w_{m}\right), \\
& \chi_{m}=u_{s}^{m}-v_{s}^{m}-\frac{r}{z}\left(w_{s}^{m}-w_{m}\right) .
\end{aligned}
$$

In addition, for all $m$,

$$
p_{m}=\frac{2}{z}\left(w_{s}^{m}-w_{m}\right)
$$

Combining (51)-(53) with the previously derived expansions of $\mathbf{u}_{s},(48)-(50)$, we thus obtain the following additional relationships among the unknown coefficients:

$$
\begin{aligned}
& E_{n}^{m} \sinh \left(n+\frac{1}{2}\right) \eta_{0}+F_{n}^{m} \cosh \left(n+\frac{1}{2}\right) \eta_{0}=X_{n}^{m}\left(\eta_{0}\right)-\frac{1}{(2 n+3) \sinh \eta_{0}}\left[-Z_{n+1}^{m}\left(\eta_{0}\right)\right. \\
& \left.+C_{n+1}^{m} \sinh \left(n+\frac{3}{2}\right) \eta_{0}\right]+\frac{1}{(2 n-1) \sinh \eta_{0}}\left[-Z_{n-1}^{m}\left(\eta_{0}\right)\right. \\
& \left.\quad+C_{n-1}^{m} \sinh \left(n-\frac{1}{2}\right) \eta_{0}\right] \quad \text { for all } m \\
& G_{n}^{0} \sinh \left(n+\frac{1}{2}\right) \eta_{0}+H_{n}^{0} \cosh \left(n+\frac{1}{2}\right) \eta_{0}=Y_{n}^{0}\left(\eta_{0}\right) \quad \text { for } \quad m=0 \\
& G_{n}^{m} \sinh \left(n+\frac{1}{2}\right) \eta_{0}+H_{n}^{m} \cosh \left(n+\frac{1}{2}\right) \eta_{0} \\
& =Y_{n}^{m}\left(\eta_{0}\right)+\frac{(n+m)(n+m+1)}{(2 n+3) \sinh \eta_{0}}\left[-Z_{n+1}^{m}\left(\eta_{0}\right)+C_{n+1}^{m} \sinh \left(n+\frac{3}{2}\right) \eta_{0}\right] \\
& -\frac{(n-m)(n-m+1)}{(2 n-1) \sinh \eta_{0}}\left[-Z_{n-1}^{m}\left(\eta_{0}\right)+C_{n-1}^{m} \sinh \left(n-\frac{1}{2}\right) \eta_{0}\right] \quad \text { for } \quad m \geqslant 1
\end{aligned}
$$




$$
\begin{aligned}
& A_{n}^{m} \sinh \left(n+\frac{1}{2}\right) \eta_{0}+B_{n}^{m} \cosh \left(n+\frac{1}{2}\right) \eta_{0}=\frac{-2}{\sinh \eta_{0}}\left[\frac { ( n - m ) } { 2 n - 1 } \left\{Z_{n-1}^{m}\left(\eta_{0}\right)\right.\right. \\
&\left.-C_{n-1}^{m} \sinh \left(n-\frac{1}{2}\right) \eta_{0}\right\}-\cosh \eta_{0}\left\{Z_{n}^{m}\left(\eta_{0}\right)-C_{n}^{m} \sinh \left(n+\frac{1}{2}\right) \eta_{0}\right\} \\
&\left.+\frac{n+m+1}{2 n+3}\left\{Z_{n+1}^{m}\left(\eta_{0}\right)-C_{n+1}^{m} \sinh \left(n+\frac{3}{2}\right) \eta_{0}\right\}\right] \text { for all } m .
\end{aligned}
$$

We have thus derived sixteen independent algebraic relations, namely $(32 a-d)$, (33)-(35), $(37 a, b),(40),(41),(44),(45),(54)-(56)$, which may be used to evaluate all of the unknown coefficients of the general solutions in the upper and lower fluids. It should be noted that these algebraic equations are all linear. Moreover, equations (44), $(45 a, b)$ and $(35 a, b)$ suggest that

$$
A_{n}^{m}=\lambda \hat{A}_{n}^{m}, \quad E_{n}^{m}=\lambda \hat{E}_{n}^{m} \quad \text { and } \quad G_{n}^{m}=\lambda \widehat{G}_{n}^{m} .
$$

The relationships (57) automatically satisfy equations (44) and $(45 a, b)$, and also satisfy $(35 a, b)$ owing to equations $(33 a, b)$. It will also be noted that $D_{n}^{m}, \hat{D}_{n}^{m}$ and $\hat{C}_{n}^{m}$ are all equal to zero according to (32) and (37). Thus, it is only necessary to solve the equations $(33 a, b),(34 a, b),(40),(41 a, b),(54),(55 a, b)$ and $(56)$ for the seven unknown coefficients: $A_{n}^{m}, B_{n}^{m}, C_{n}^{m}, E_{n}^{m}, F_{n}^{m}, G_{n}^{m}$ and $H_{n}^{m}$. This system of algebraic equations yields a band matrix and is readily solved using standard numerical methods for specified values of $\eta_{0}$ (i.e. of particle position relative to the interface). Once the coefficients have been determined for the lower fluid, the coefficients for the upper fluid can be obtained trivially from equations (57) and (32).

\section{An alternative method for axisymmetric flow}

When the sphere is translating normal to the (plane) interface, the flow field can also be obtained using a stream function representation of the governing equations. Stimson \& Jeffery (1926) derived a solution for the stream function in bipolar coordinates in order to solve for the motion generated by two spheres which are translating with equal constant velocities parallel to their line of centres. Bart (1968) later utilized the same stream function solution to evaluate the drag force for a spherical drop which is moving normal to a flat fluid/fluid interface. However, Bart (1968) only reported the sum of the coefficients in the stream function solution which yield the drag. Therefore, for completeness, we will briefly discuss the use of the stream function solution for axisymmetric flow and present the values of all the coefficients in the stream function solution for translation of a solid sphere perpendicular to a plane fluid/fluid interface.

The stream function for any axisymmetric Stokes' flow is well known to satisfy the general equation

$$
E^{4} \psi=0
$$

When expressed in terms of a cylindrical co-ordinate system, with the $z$ axis being the axis of symmetry,

$$
\begin{gathered}
E^{2} \equiv r \frac{\partial}{\partial r}\left(\frac{1}{r} \frac{\partial}{\partial r}\right)+\frac{\partial^{2}}{\partial z^{2}}, \\
u=\frac{1}{r} \frac{\partial \psi}{\partial z} \quad \text { and } \quad w=-\frac{1}{r} \frac{\partial \psi}{\partial r} .
\end{gathered}
$$


Stimson \& Jeffery's (1926) general solution of equation (58) may be expressed in the form

where

$$
\psi=(\cosh \eta-\zeta)^{-\frac{3}{2}} \sum_{n} U_{n} V_{n}
$$

$$
\begin{aligned}
& U_{n}=K_{n} \cosh \left(n-\frac{1}{2}\right) \eta+L_{n} \sinh \left(n-\frac{1}{2}\right) \eta+M_{n} \cosh \left(n+\frac{3}{2}\right) \eta+N_{n} \sinh \left(n+\frac{3}{2}\right) \eta \\
& V_{n}=P_{n-1}(\zeta)-P_{n+1}(\zeta) .
\end{aligned}
$$

Now, let us suppose that $\psi$ represents the stream function for the lower fluid. Then the stream function for the upper fluid, $\hat{\psi}$, will have the same functional form with coefficients, $\hat{K}_{n}, \hat{L}_{n}, \hat{M}_{n}$ and $\hat{N}_{n}$. Applying the boundary conditions that were discussed in the previous section, we can evaluate the coefficients $K_{n}, \ldots, N_{n}, \widehat{K}_{n}, \ldots, \widehat{N}_{n}$ for translation of a sphere normal to a plane fluid/fluid interface. The viscosity ratio between two fluids is again denoted by $\lambda$ and the sphere is located at $\eta=\eta_{0}$. The results are

$$
\begin{gathered}
K_{n}=-M_{n}=\lambda \hat{K}_{n}, \\
\hat{K}_{n}=-\hat{L}_{n}=-\hat{M}_{n}=\hat{N}_{n}, \\
K_{n}=\frac{\lambda}{2}\left[\left(n-\frac{1}{2}\right) L_{n}+\left(n+\frac{3}{2}\right) N_{n}\right], \\
L_{n}=(2 n+3) k_{n}\left[\frac{-\left(a_{n}+b_{n}\right)+\left(c_{n}+d_{n}\right) \lambda+(2 n-1)(2 n+1) \sinh ^{2} \eta_{0}}{b_{n}-\lambda d_{n}}\right], \\
N_{n}=(2 n-1) k_{n}\left[\frac{\left(a_{n}+b_{n}\right)-\left(c_{n}+d_{n}\right) \lambda-(2 n+1)(2 n+3) \sinh ^{2} \eta_{0}}{b_{n}-\lambda d_{n}}\right] .
\end{gathered}
$$

Here,

$$
\begin{aligned}
& k_{n}=\frac{n(n+1) \sinh ^{2} \eta_{0}}{\sqrt{2(2 n-1)(2 n+1)(2 n+3)}}, \\
& a_{n}=(2 n+1)^{2} \sinh ^{2} \eta_{0}+4 \cosh ^{2}\left(n+\frac{1}{2}\right) \eta_{0}, \\
& b_{n}=2 \sinh (2 n+1) \eta_{0}-(2 n+1) \sinh 2 \eta_{0}, \\
& c_{n}=2 \sinh (2 n+1) \eta_{0}+(2 n+1) \sinh 2 \eta_{0}, \\
& d_{n}=4 \sinh ^{2}\left(n+\frac{1}{2}\right) \eta_{0}-(2 n+1)^{2} \sinh \eta_{0} .
\end{aligned}
$$

The drag force on the sphere can be easily derived (cf. Stimson \& Jeffery 1926):

$$
\begin{aligned}
F_{z} & =\frac{\sqrt{ } 2}{3} \operatorname{cosech} \eta_{0} \sum_{n}\left(K_{n}+L_{n}+M_{n}+N_{n}\right) \\
& =\frac{4 \sqrt{2}}{3} \operatorname{cosech} \eta_{0} \sum_{n} k_{n}\left[\frac{-a_{n}+\lambda c_{n}}{b_{n}-\lambda d_{n}}-1\right] .
\end{aligned}
$$

Here $F_{z}$ is non-dimensionalized with respect to $6 \pi \mu a U$. Equation (66) is given by Bart (1968). 


\section{Translation and/or rotation of a rigid sphere in a quiescent fluid near a plane fluid interface}

Let us now consider the specific problem of a rigid sphere which is translating or rotating in a quiescent fluid near a plane fluid/fluid interface. This problem may be solved directly, for an arbitrary direction of translation or rotation, using the methods outlined in $\S 2$. All that is required is a specification of the surface velocity of the sphere in terms of bipolar eigensolutions; namely, the coefficients $X_{n}^{m}, Y_{n}^{m}$ and $Z_{n}^{m}$ in (48)-(50), and solution of the resulting algebraic relationships. Owing to the linearity of Stokes' equation and boundary conditions in the case of a flat interface, however, the velocity and pressure fields generated by any arbitrary motion of the sphere can be obtained by superposing the fields associated with only four fundamental modes of sphere motion; namely, translation perpendicular and parallel to the interface, and rotation (with the axis of rotation) perpendicular and parallel to the interface. Indeed, we have shown in part 1 how the force and torque on the particle can be related to the translational and angular velocities of the particle via three independent second-order resistance tensors whose components can be determined completely by considering the same set of flows. In the present section, we therefore consider application of the general techniques and solution of $\S 2$ to obtain exact solutions for the four fundamental problems mentioned above. We will consider both the detailed pressure and velocity fields, and the hydrodynamic force and torque on the sphere. As indicated in the introduction to this paper, we shall be particularly concerned with the comparison between the exact results obtained here, and the asymptotic results of part 1 .

We assume, in the following, that the sphere is centred at $z=-l$ (note that $l=1$ corresponds to the sphere just touching the interface). Thus, the sphere surface is represented by $\eta=\eta_{0}=-\cosh ^{-1} l$, and the constant $c$ in the co-ordinate transformation, equation (5), is given as $c=\left(l^{2}-1\right)^{\frac{1}{2}}$. The hydrodynamic force and torque on the particle can be calculated directly from the stress at the particle surface

$$
\begin{aligned}
& \mathbf{F}=\iint d \mathbf{s . t}, \\
& \mathbf{T}=\iint \mathbf{r} \times(d \mathbf{s} . \mathbf{t}) .
\end{aligned}
$$

Here, $\mathbf{t}$ is the stress tensor, and $\mathbf{r}$ is the position vector of a surface element relative to the sphere centre. The drag force and hydrodynamic torque may be non-dimensionalized with respect to $F_{c}=6 \pi \mu a U$ (or $6 \pi \mu a^{2} \Omega$ ) and $T_{c}=8 \pi \mu a^{2} U$ (or $8 \pi \mu a^{3} \Omega$ ), where $U$ and $\Omega$ represent the magnitudes of the translational and rotational velocity, respectively. In the following discussion, we shall refer to the force for translation and the torque for rotation, non-dimensionalized in this fashion, as the drag ratio and the torque ratio, respectively, since they are scaled with the force and torque which would act on the sphere in an infinite fluid.

\subsection{Boundary conditions for the four fundamental problems}

Let us now turn to the specific boundary conditions, as well as formulae for the (non-dimensionalized) hydrodynamic force and/or torque on the sphere for the four fundamental modes of particle motion.

Case (i). Translation of a non-rotating sphere perpendicular to a plane interface. First, we consider the translation of a non-rotating rigid sphere perpendicular to the plane 
fluid interface. In view of the axisymmetric nature of the problem, it is clear that the solution must be independent of the azimuthal angle, $\phi$, so that the only non-zero coefficients in the general solution of $\S 2$ are those with $m=0$. In addition $\alpha_{0}=0$.

The remaining constants can be determined from the prescribed velocity of the sphere

$$
u_{s}=v_{s}=0, \quad w_{s}=1 \quad \text { on } \eta=\eta_{0} .
$$

Expanding $w_{s}=1$ in the form (50), the constants $X_{n}^{0}, Y_{n}^{0}$ and $Z_{n}^{0}$, which appear in $(54)-(56)$, are easily seen to be

$$
\left.\begin{array}{rl}
X_{n}^{0} & =Y_{n}^{0}=0, \\
Z_{n}^{0} & =\sqrt{ } 2 e^{\left(n+\frac{1}{2}\right) \eta_{0}}
\end{array}\right\}
$$

The constants $A_{n}^{0}, B_{n}^{0}, \ldots, \hat{H}_{n}^{0}$ can thus be evaluated in the manner outlined in $\S 2.2$.

It can be shown, after a tedious algebraic manipulation (cf. Dean \& O'Neill 1963) starting from equation (67), that

$$
\left.\begin{array}{l}
F_{x}=F_{y}=0, \\
F_{z}=\frac{-\sqrt{ } 2}{3} \sinh \eta_{0} \sum_{n}\left[C_{n}^{0}-\left(n+\frac{1}{2}\right)\left(A_{n}^{0}-B_{n}^{0}\right)\right],
\end{array}\right\}
$$

while the torque is identically zero

as expected.

$$
\mathbf{T}=\mathbf{0}
$$

Case (ii). Translation of a non-rotating sphere parallel to a plane interface. We now turn to the problem of a sphere translating in the $\mathbf{i}_{x}$ direction. In this case, all terms in the general solution of $\S 2$ vanish except for those with $m=1$, and $\alpha_{1}=0$. On the sphere surface,

$$
u_{s}=\cos \phi, \quad v_{s}=-\sin \phi \quad \text { and } \quad w_{s}=0 .
$$

In consequence, $X_{n}^{1}, Y_{n}^{1}$ and $Z_{n}^{1}$ are

$$
\left.\begin{array}{l}
X_{n}^{1}=Z_{n}^{1}=0 \\
Y_{n}^{1}=2 \sqrt{ } 2 e^{\left(n+\frac{1}{2}\right) \eta_{0}},
\end{array}\right\}
$$

and the unknown coefficients can be determined as described above.

The drag force and hydrodynamic torque, in this case, are related to these constant coefficients by means of the formulae (cf. O'Neill 1964)

and

$$
\left.\begin{array}{l}
F_{y}=F_{z}=0 \\
F_{x}=\frac{-\sqrt{ } 2}{6} \sinh \eta_{0} \sum_{n}\left[G_{n}^{1}-H_{n}^{1}+n(n+1)\left(A_{n}^{1}-B_{n}^{1}\right)\right],
\end{array}\right\}
$$

$$
\left.\begin{array}{rl}
T_{x}= & T_{z}=0 \\
T_{y}= & \frac{\sinh ^{2} \eta_{0}}{12 \sqrt{ } 2} \sum_{n}\left[( 2 + e ^ { ( 2 n + 1 ) \eta _ { 0 } } ) \left\{n(n+1)\left(-2 C_{n}^{1}-A_{n}^{1} \operatorname{coth} \eta_{0}\right)\right.\right. \\
& \left.-\left(2 n+1+\operatorname{coth} \eta_{0}\right) G_{n}^{1}\right\}+\left(2-e^{(2 n+1) \eta_{0}}\right)\left\{n(n+1) B_{n}^{1} \operatorname{coth} \eta_{0}\right. \\
& \left.\left.+\left(2 n+1+\operatorname{coth} \eta_{0}\right) H_{n}^{1}\right\}\right] .
\end{array}\right\}
$$

Case (iii). Rotation of a sphere normal to a plane interface. The third problem which 
we consider is the rotation of a sphere with the axis of rotation normal to a plane interface. In the general solution of Stokes' equation, the non-zero terms in this case are for $m=0$ and $\alpha_{0}=\frac{1}{2} \pi$. Moreover, $p, u$ and $w$ are identically zero, as is obvious from the symmetry of the problem. We can thus calculate the coefficients for $v$ analy tically, rather than numerically. From equations (32), (41a) and (57),

$$
\hat{G}_{n}^{0}=-\hat{H}_{n}^{0}, \quad H_{n}^{0}=\hat{H}_{n}^{0} \quad \text { and } \quad G_{n}^{0}=\lambda \hat{G}_{n}^{0} .
$$

The boundary condition on the sphere surface is

$$
v_{s}=r \text { on } \eta=\eta_{0} .
$$

Therefore, from equations $(47 b)$ and (49a), it can be shown that $Y_{n}^{0}$ takes the value

$$
Y_{n}^{0}=2 \sqrt{ } 2 c e^{\left(n+\frac{1}{2}\right) \eta_{0}} \text {. }
$$

The constants $G_{n}^{0}$ and $H_{n}^{0}$ can then be obtained from equations (55a), (77) and (79):

$$
\begin{aligned}
G_{n}^{0} & =\frac{2 \sqrt{ } 2 c \lambda e^{\left(n+\frac{1}{2}\right) \eta_{0}}}{\lambda \sinh \left(n+\frac{1}{2}\right) \eta_{0}-\cosh \left(n+\frac{1}{2}\right) \eta_{0}}, \\
H_{n}^{0} & =-\frac{1}{\lambda} G_{n}^{0} .
\end{aligned}
$$

The remaining non-zero constants follow from (77). It may be seen from (67) that the drag force is identically zero:

$$
\mathbf{F}=0 \text {, }
$$

while the hydrodynamic torque (cf. Jeffery 1915) is

$$
\left.\begin{array}{l}
T_{x}=T_{y}=0 \\
T_{z}=\frac{\sinh ^{2} \eta_{0}}{\sqrt{ } 2} \sum_{n} n(n+1)\left(-G_{n}^{0}+H_{n}^{0}\right) \cdot
\end{array}\right\}
$$

Case (iv). Rotation of a sphere parallel to a plane interface. Finally, let us consider a rotating sphere whose rotation axis is parallel to the $y$ axis. The boundary conditions on the sphere surface in this case are

$$
u_{s}=(z+l) \cos \phi, \quad v_{s}=-(z+l) \sin \phi \quad \text { and } \quad w_{s}=-r \cos \phi .
$$

Thus, the non-zero terms in the general solution are only those for $m=1$ and $\alpha_{1}=0$. $X_{n}^{1}, Y_{n}^{1}$ and $Z_{n}^{1}$ can be evaluated from the equations (84), $(48 b),(49 b)$ and $(50)$ :

$$
\left.\begin{array}{l}
X_{n}^{1}=0 \\
Y_{n}^{1}=-2 \sqrt{ } 2 c\left(2 n+1+\operatorname{coth} \eta_{0}\right) e^{\left(n+\frac{1}{2}\right) \eta_{0}}, \\
Z_{n}^{1}=-2 \sqrt{ } 2 c e^{\left(n+\frac{1}{2}\right) \eta_{0}} .
\end{array}\right\}
$$

The drag force and hydrodynamic torque are calculated from the equations (67) and (68) (cf. Dean \& O’Neill 1963):

$$
\left.\begin{array}{l}
F_{x}=\frac{-\sqrt{ } 2}{6} \sinh \eta_{0} \sum_{n}\left[G_{n}^{1}-H_{n}^{\mathrm{x}}+n(n+1)\left(A_{n}^{1}-B_{n}^{1}\right)\right], \\
F_{y}=F_{z}=0,
\end{array}\right\}
$$




$$
\left.\begin{array}{rl}
T_{x}= & T_{z}=0, \\
T_{y}= & -\frac{1}{3}-\frac{\sinh ^{2} \eta_{0}}{12 \sqrt{2}} \sum_{n}\left[\left(2+e^{(2 n+1) \eta_{0}}\right)\right. \\
& \times\left\{n(n+1)\left(-2 C_{n}^{1}-A_{n}^{1} \operatorname{coth} \eta_{0}\right)\right. \\
& \left.-\left(2 n+1+\operatorname{coth} \eta_{0}\right) G_{n}^{1}\right\} \\
& \left.+\left(2-e^{(2 n+1) \eta_{0}}\right)\left\{n(n+1) B_{n}^{1} \operatorname{coth} \eta_{0}+\left(2 n+1+\operatorname{coth} \eta_{0}\right) H_{n}^{1}\right\}\right] .
\end{array}\right\}
$$

\subsection{Numerical results and discussion}

In $\S 4.1$ we have derived formulae from which the velocity fields, and the hydrodynamic force and torque on the sphere, can be calculated for the four fundamental modes of motion of a sphere near a flat fluid interface; namely, translation perpendicular and parallel to the interface, and rotation with the axis of rotation parallel and perpendicular to the interface. With the exception of the last problem, which was solved analytically, the unknown coefficients in the general solution for the two fluids must be obtained via a numerical solution for the infinite set of governing algebraic equations that were outlined earlier. From equations (70), (74), (79) and (85), however, it is evident that the coefficients for large $n$ will decrease in magnitude as $e^{\left(n+\frac{1}{2}\right) \eta_{0}}$, where $\eta_{0}=-\cosh ^{-1} l$. Thus, when $l \gg 1$, the coefficients decrease quickly (exponentially) in magnitude as the index $n$ is increased. In this case, a very good approximation can be obtained by truncating the solution series for ' large' $n$. The resulting set of equations is finite and banded, and can be solved very efficiently using standard Gaussian elimination schemes for band matrices. However, as $l \rightarrow 1$, the rate of decrease of the coefficients with increasing $n$ becomes slower, and it is necessary to include increasing numbers of terms (i.e. larger values of $n$ ) in order to give the same numerical accuracy in the results. Since the coefficients decrease monotonically with increase of $n$ (for any fixed value of $l$ ) after the first few terms, it is relatively easy to estimate the numerical magnitude of the error which is caused by truncation. The numerical error in the calculation of coefficients due to the truncation of terms with $n>n_{\max }$ is analysed in the appendix. Once we calculate the coefficients, we can evaluate the contribution to the drag force and hydrodynamic torque from the terms in the series for each $n$. The magnitude of these terms becomes approximately a geometrical series for large $n$. Thus, we can easily estimate the numerical magnitude of error in the calculated values of drag force and hydrodynamic torque due to truncation. To limit the maximum relative error in the computed results for the drag force and hydrodynamic torque to values less than $5 \times 10^{-7}$, we used $n_{\max }=10$ for $l \geqslant 3 \cdot 0, n_{\max }=15$ for $3.0>l \geqslant 1.8, n_{\max }=25$ for $1.8>l \geqslant 1.2$ and $n_{\max }=30$ for $1.2>l \geqslant 1.1$.

In this section, we will present and discuss the results calculated for the velocity and pressure fields, as well as for the drag and torque ratios for the four problems listed above. Particularly significant is the resulting comparison of the present 'exact' solutions with the approximate solutions for $l \gg 1$ which were obtained in part 1 of this work. When the sphere is very close to the interface, on the other hand, so that $l-1 \ll 1$, lubrication theory can be applied, in principle, to obtain asymptotic solutions. Indeed, Goldman, Cox \& Brenner (1967) used this technique to study the translation and rotation of a sphere near a plane solid wall. However, they found that the lubrication-theory results for force and/or torque were quite poor when compared 
with the numerically exact results of Dean \& O'Neill (1963) and O'Neill (1964). An explanation for this discrepancy was given by O'Neill \& Stewartson (1967), who independently investigated the translation of a sphere parallel to a plane solid wall, using lubrication theory. These authors showed that lubrication theory does provide an accurate description of local flow properties, but that it cannot be used (without extension) for prediction of properties of the overall flow, such as force or torque, with any degree of reliability if the flow domain includes substantial regions of weakly sheared flow away from the lubrication gap. Although O'Neill \& Stewartson were eventually able to obtain successful asymptotic results for $(l-1) \ll 1$, the analysis required an elaborate matching procedure to generate a solution in the 'outer' region away from the gap. No such study has yet been completed for analysing the motion of a particle which is very close to a fluid/fluid interface. Therefore, at the present time, the only results available for $(l-1) \ll 1$ which could be compared with the exact solutions of this paper are for the case of solid wall, i.e. $\lambda=\infty$. Such a comparison has already been reported by O'Neill \& Stewartson (1967) using the results of O'Neill (1964), and will thus not be repeated here.

We begin, in table 1, with the drag ratio for translation perpendicular to the plane interface calculated by means of our general solution technique. This same quantity was previously calculated by Bart (1968) using the stream function formulation which was discussed in $\S 3$, and was also recalculated by us using this latter approach. Our calculations using the stream function expansion agreed exactly with the results of Bart (1968) when compared at the same value of $l$ (it may be noted here that Bart used incremental values of $\eta_{0}$ and thus obtained different values of $l$ from those listed in table 1). Further, the results calculated via the general solution were found to agree exactly with the values obtained by the stream function expansion. In figure 2 , the 'exact' drag ratios obtained in the present work are compared, for $\lambda=0,1$ and $\infty$, with these obtained using the approximate method of part 1 . Both results show that the drag ratio increases rapidly as the sphere approaches the interface, for all $\lambda$, owing to the assumption of a flat interface. However, the drag ratio obtained in part 1 does not increase as fast as the exact result in the limit as $l$ approaches unity; i.e. as the sphere approaches the plane interface. The drag ratio from the 'exact' solution is, in fact, unbounded as $l \rightarrow 1$. The approximate expansion, on the other hand, has $l^{-1}$ as the 'small' parameter and is, therefore, strictly valid only when the sphere is far from the interface. In fact, the approximate solution including terms through $O\left(l^{-2}\right)$ is seen, from figure 2 , to represent the drag ratio to within $10 \%$ for $l$ as small as 2 . It cannot under any circumstances exhibit the singular behaviour of the exact solution for $l \rightarrow 1$. Nevertheless, the approximate solution does give a remarkably accurate representation of the exact result, over almost the whole range of possible sphere positions. This is important, as suggested in $\S 1$, because it is the approximate solution scheme which can be generalized to other particle shapes which may be important in applications. The eigensolution expansion is useful for general motions only for the case of a sphere which is considered here.

For the case of a sphere translating, without rotation, parallel to the interface, we have numerically evaluated the drag ratio, as well as the hydrodynamic torque on the sphere. The latter is equal in magnitude but opposite in sense to the torque which must be applied to the particle by external means to keep it from rotating. The drag ratio is given as a function of the position of the sphere in table 2. Examination shows 


\begin{tabular}{|c|c|c|c|c|c|c|}
\hline$l$ & $\lambda$ & 0 & 0.1 & 1 & 10 & $\infty$ \\
\hline $10 \cdot 0$ & & $1 \cdot 08096$ & 1.08492 & $1 \cdot 10311$ & $1 \cdot 12193$ & $1 \cdot 12619$ \\
\hline $5 \cdot 0$ & & $1 \cdot 17560$ & $1 \cdot 18477$ & 1.22788 & $1 \cdot 27429$ & $1 \cdot 28509$ \\
\hline $\mathbf{3 \cdot 0}$ & & 1.33015 & $1 \cdot 34875$ & 1.43954 & 1.54387 & 1.56921 \\
\hline $2 \cdot 0$ & & $1 \cdot 59668$ & 1.63314 & 1.82163 & $2 \cdot 06257$ & $2 \cdot 12554$ \\
\hline 1.8 & & $1 \cdot 71575$ & $1 \cdot 76053$ & 1.99726 & $2 \cdot 31347$ & $2 \cdot 39877$ \\
\hline $1 \cdot 6$ & & 1.90031 & 1.95812 & $2 \cdot 27341$ & $2 \cdot 72202$ & $2 \cdot 84891$ \\
\hline 1.4 & & $2 \cdot 23492$ & $2 \cdot 31625$ & $2 \cdot 78090$ & 3.51180 & $3 \cdot 73562$ \\
\hline $1 \cdot 2$ & & $3 \cdot 10459$ & $3 \cdot 24343$ & $4 \cdot 10294$ & $5 \cdot 74513$ & 6.34088 \\
\hline $1 \cdot 1$ & & $4 \cdot 62554$ & 4.85033 & $6 \cdot 34941$ & $9 \cdot 88127$ & $11 \cdot 45916$ \\
\hline
\end{tabular}

TABLE 1. Drag ratio for a sphere translating perpendicular to a plane interface.

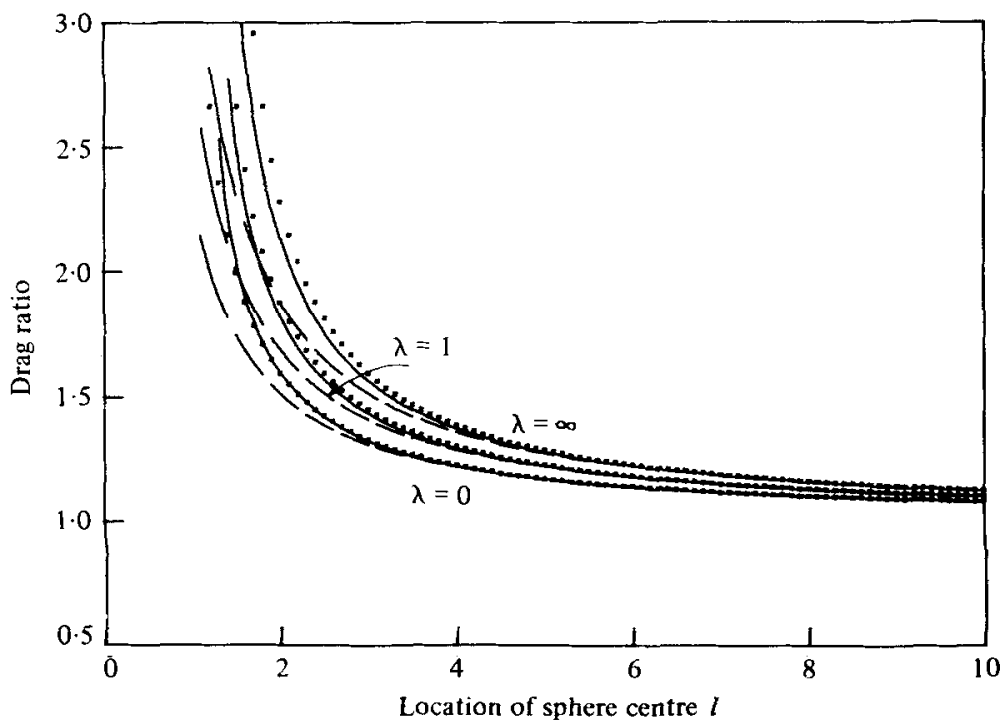

FIGURe 2. Drag ratio for the translation of a sphere perpendicular to a plane interface, - exact solution; - - , approximate solution; $\mathbf{\square} \mathbf{\square}$, summed series.

that the drag actually decreases relative to Stokes' drag for an unbounded fluid for $\lambda=0$ and $0 \cdot 1$. On the other hand, for the larger values of $\lambda=1,10$ and $\infty$, the drag increases relative to that in unbounded fluid as the sphere comes closer to the interface, at a rate which increases with increasing $\lambda$. These results are in qualitative accord with the asymptotic solution, as may be seen in figure 3 . Indeed, in this case, the asymptotic and 'exact' predictions for the drag ratio agree within $5 \%$ up to $l=1 \cdot 1$ for $\lambda=0$ and $\lambda=1$, and to $l \sim 1.8$ for $\lambda=\infty$. The somewhat poorer performance of the asymptotic solution in the latter case is again a reflexion of the fact that the exact result for the drag ratio is singular in the limit $l \rightarrow 1$. The hydrodynamic torque which acts on the translating particle was also calculated as a function of $\lambda$ and $l$, and the detailed numerical results are shown in table 3 . In addition, the numerical results for $\lambda=0,1$ and $\infty$ are compared with the approximate solution in figure 4 . There is reasonable qualitative agreement between the two for $l>1 \cdot 1$ in the cases $\lambda=0$ and $\lambda=1$. For $\lambda=\infty$, on the other hand, the asymptotic prediction, to $O\left(l^{-2}\right)$, is that the 


\begin{tabular}{|c|c|c|c|c|c|c|}
\hline$l$ & $\lambda$ & 0 & $0 \cdot 1$ & 1 & 10 & $\infty$ \\
\hline $10 \cdot 0$ & & 0.963802 & 0.971778 & 1.00937 & 1.05000 & $1 \cdot 05948$ \\
\hline $5 \cdot 0$ & & 0.929866 & 0.944792 & 1.01843 & $1 \cdot 10466$ & $1 \cdot 12586$ \\
\hline $3 \cdot 0$ & & 0.887536 & 0.910258 & 1.02935 & $1 \cdot 18558$ & $1 \cdot 22716$ \\
\hline $2 \cdot 0$ & & $0 \cdot 838826$ & 0.869082 & $1 \cdot 04038$ & $1 \cdot 30374$ & $1 \cdot 38275$ \\
\hline $1 \cdot 8$ & & 0.823629 & 0.855875 & $1 \cdot 04331$ & $1 \cdot 34899$ & 1.44521 \\
\hline $1 \cdot 6$ & & 0.805414 & 0.839792 & $1 \cdot 04641$ & 1.41140 & $1 \cdot 53438$ \\
\hline $1 \cdot 4$ & & 0.783367 & 0.819922 & 1.04946 & 1.50476 & $1 \cdot 67553$ \\
\hline $1 \cdot 2$ & & 0.756617 & 0.795140 & $1 \cdot 05172$ & $1 \cdot 66838$ & 1.95271 \\
\hline $1 \cdot 1$ & & $0 \cdot 741287$ & 0.780568 & $1 \cdot 05191$ & $1 \cdot 82067$ & $2 \cdot 26430$ \\
\hline
\end{tabular}

TABLE 2. Drag ratio for a sphere translating parallel to a plane interface.

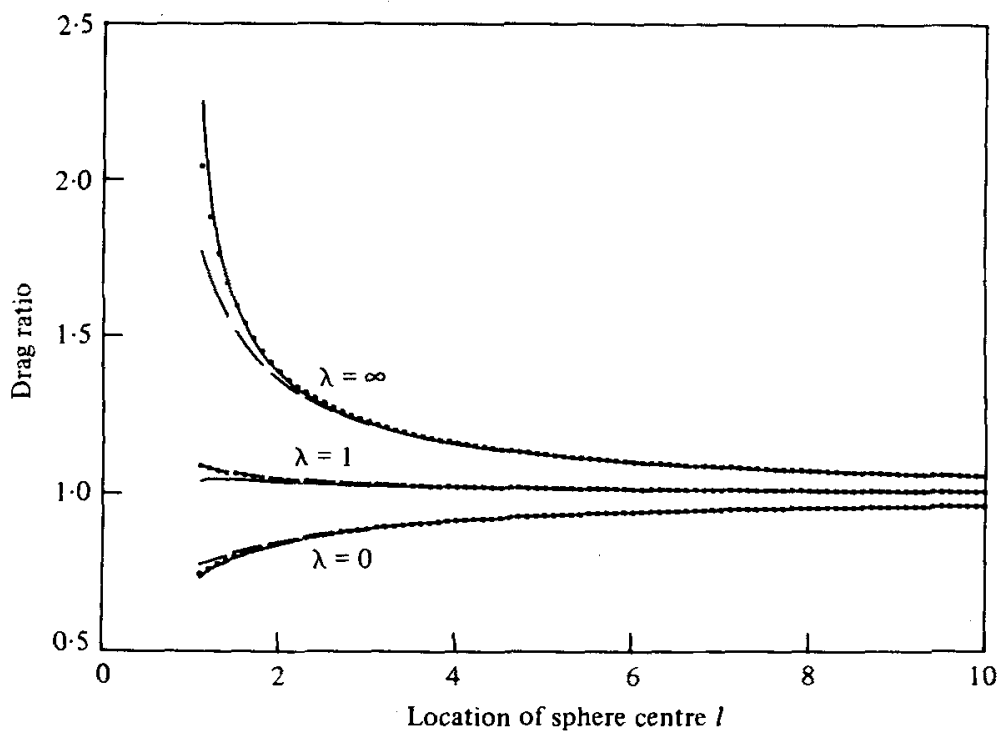

Figure 3. Drag ratio for the translation of a sphere parallel to a plane interface. $\longrightarrow$, exact solution; - - , approximate solution; $\square \square$, summed series.

torque is identically equal to zero, while the exact numerical result shows a rapid increase in the magnitude of the torque as the sphere approaches the interface. The asymptotic result is consistent with the analysis of Faxen (1921) who found that the leading contribution to the torque for a plane solid wall is $O\left(l^{-4}\right)$. The exact solution shows that the torque for $\lambda=\infty$ only becomes significant for $l<\sim 2 \cdot 5$, whereas the torque for the smaller values of $\lambda$ is noticeable for $l \sim 6-7$, and these observations are again suggestive that the wall effect will appear as a higher-order contribution in the asymptotic framework of part 1 . The fact that this wall contribution becomes large as $l \rightarrow 1$ serves as a reminder that higher-order terms in the asymptotic expansion of part 1 do not necessarily remain small when the expansion is pushed beyond its natural range of applicability.

It will be noted, either from table 3 or figure 4 , that the torque has a different sign in the limit of a solid wall $(\lambda=\infty)$, than it does for a free interface $(\lambda=0)$. In the former case, the sphere would rotate, in the absence of an applied torque, in a direction 


\begin{tabular}{|c|c|c|c|c|c|}
\hline$l$ & $\lambda$ & $0 \cdot 1$ & 1 & 10 & $\infty$ \\
\hline $10 \cdot 0$ & $1.80701 \times 10^{-3}$ & $1.65564 \times 10^{-3}$ & $9 \cdot 42116 \times 10^{-4}$ & $1.70909 \times 10^{-4}$ & $-9.01197 \times 10^{-6}$ \\
\hline $5 \cdot 0$ & $6.97030 \times 10^{-3}$ & $6.42965 \times 10^{-3}$ & $3.76051 \times 10^{-3}$ & $6.31416 \times 10^{-4}$ & $-1.38502 \times 10^{-4}$ \\
\hline $3 \cdot 0$ & $1.84409 \times 10^{-2}$ & $1.71498 \times 10^{-2}$ & $1.03518 \times 10^{-2}$ & $1.37629 \times 10^{-3}$ & $-1.02023 \times 10^{-3}$ \\
\hline $2 \cdot 0$ & $3.89 t 04 \times 10^{-2}$ & $3.65666 \times 10^{-2}$ & $2.29995 \times 10^{-2}$ & $1.51380 \times 10^{-3}$ & $-5.02471 \times 10^{-3}$ \\
\hline $1 \cdot 8$ & $4.69457 \times 10^{-z}$ & $4.42916 \times 10^{-2}$ & $2 \cdot 83220 \times 10^{-2}$ & $1.07144 \times 10^{-3}$ & $-7.68745 \times 10^{-3}$ \\
\hline $1 \cdot 6$ & $5.76360 \times 10^{-2}$ & $5.46761 \times 10^{-2}$ & $3.58197 \times 10^{-2}$ & $-5 \cdot 26852 \times 10^{-3}$ & $-1.25431 \times 10^{-2}$ \\
\hline $1 \cdot 4$ & $7 \cdot 21370 \times 10^{-2}$ & $6.90015 \times 10^{-2}$ & $4.70316 \times 10^{-2}$ & $-2.83454 \times 10^{-3}$ & $-2 \cdot 26136 \times 10^{-2}$ \\
\hline $1 \cdot 2$ & $9.20012 \times 10^{-2}$ & $8.92500 \times 10^{-2}$ & $6.56741 \times 10^{-2}$ & $-1.04024 \times 10^{-2}$ & $-4.96377 \times 10^{-2}$ \\
\hline $1 \cdot 1$ & $1.04431 \times 10^{-1}$ & $1 \cdot 02479 \times 10^{-1}$ & $8.11697 \times 10^{-2}$ & $-1.92084 \times 10^{-2}$ & $-8.87633 \times 10^{-2}$ \\
\hline \multicolumn{6}{|c|}{$\begin{array}{c}\text { Table 3. Hydrodynamic torque, } T_{y} \text {, scaled with respect to }\left[8 \pi \mu U a^{2}\right] \text {, on a } \\
\text { sphere translating parallel to a plane interface. }\end{array}$} \\
\hline
\end{tabular}

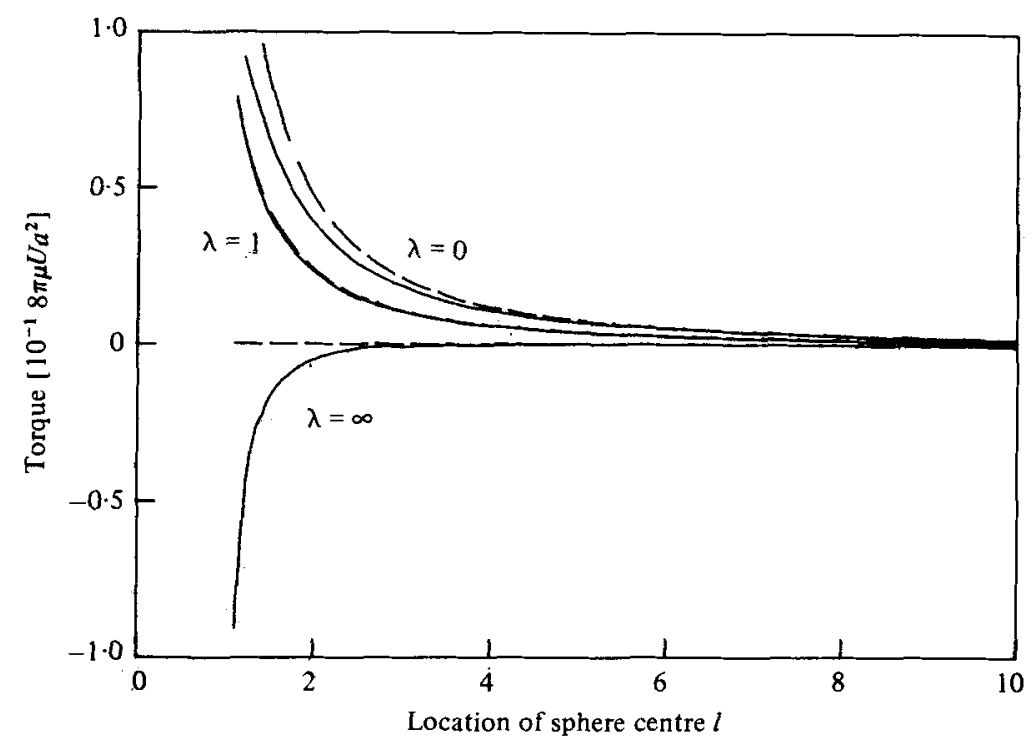

FIGURE 4. Hydrodynamic torque, scaled with respect to $\left[8 \pi \mu U a^{2}\right]$, on a sphere translating parallel to a plane interface. - - exact solution; - - - approximate solution.

consistent with 'rolling' along the wall. For the free surface, on the other hand, the sphere is predicted to rotate in the opposite direction. Moreover, it may be seen from table 3 that the torque actually changes sign for intermediate $\lambda$ as the sphere comes from a large distance inward toward the interface. The sense of the induced (hydrodynamic) torque in the 'rolling' mode is established primarily as a consequence of the fact that a much more viscous fluid above the interface yields a small slip velocity on the interface and thus higher velocity gradients above the sphere than below it. The 'reversal' in the induced torque when the upper fluid is much less viscous than the lower fluid results primarily from the existence of a substantial slip velocity on the interface, and a resultant velocity gradient above the sphere which is smaller than below. The fact that the interface remains flat in the present theory, does not play a critical role in this aspect of the parallel-translation problem. A more detailed examination of the sense of the induced torque, or, equivalently, of the direction of the rotation which would occur in the absence of an applied torque, is presented in 


\begin{tabular}{|c|c|c|c|c|c|c|}
\hline$l$ & $\lambda$ & 0 & $0 \cdot 1$ & 1 & 10 & $\infty$ \\
\hline $10 \cdot 0$ & & 0.999875 & $0 \cdot 999898$ & $1 \cdot 00000$ & 1.00010 & 1.00013 \\
\hline $5 \cdot 0$ & & 0.999001 & 0.999183 & 1.00000 & $1 \cdot 00082$ & 1.00100 \\
\hline $3 \cdot 0$ & & 0.995394 & 0.996228 & 1.00000 & 1.00380 & 1.00465 \\
\hline $2 \cdot 0$ & & 0.984666 & 0.987411 & 1.00000 & $1 \cdot 01299$ & 1.01593 \\
\hline $1 \cdot 8$ & & 0.979135 & 0.982846 & 1.00000 & 1.01794 & 1.02203 \\
\hline $1 \cdot 6$ & & 0.970698 & 0.975851 & 1.00000 & $1 \cdot 02585$ & 1.03184 \\
\hline $1 \cdot 4$ & & 0.957358 & 0.964702 & 1.00000 & $1 \cdot 03950$ & 1.04893 \\
\hline $1 \cdot 2$ & & 0.935753 & 0.946358 & $1 \cdot 00000$ & 1.06624 & $1 \cdot 08322$ \\
\hline $1 \cdot 1$ & & 0.920464 & 0.933118 & $1 \cdot 00000$ & 1.09149 & $1 \cdot 11707$ \\
\hline
\end{tabular}

TABLE 4. Torque ratio for a rotating sphere when the axis of rotation is normal to a plane interface.

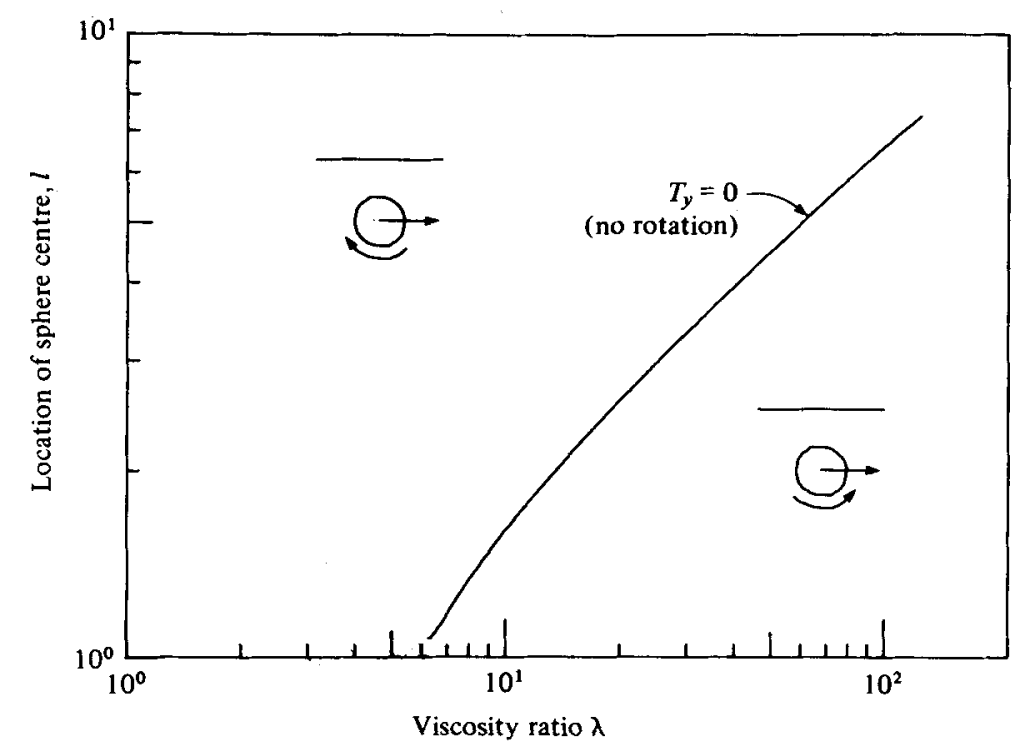

Frgure 5. The location of sphere centre where $T_{v}=0$ for translation of a sphere parallel to a plane interface.

figure 5. Here, we have plotted the position of the sphere centre where the induced hydrodynamic torque is identically zero, as a function of the viscosity ratio, $\lambda$. As $\lambda$ increases, we see that the location of this point moves farther from the interface. The fact that $\lambda_{\text {crit }} \rightarrow \sim 6$, as $l \rightarrow 1$ is somewhat surprising, and not easily explainable.

The problem of a rotating sphere whose rotation axis is normal to the interface is, as previously mentioned, easy to analyse because there is only one non-zero velocity component, $v$, which is parallel to the flat interface. Since the condition of zero normal velocity at the interface is satisfied identically in this case, the velocity field and the torque required to rotate the sphere will only differ from the case of rotation in a single unbounded fluid if the viscosities of the two fluids are not equal, i.e. if $\lambda \neq 1$. Furthermore, it is evident that the torque ratio must be larger than 1 if $\lambda>1$, and smaller than 1 if $\lambda<1$. In table 4 , the torque ratios are tabulated for various values of $\lambda$ and, in figure 6, they are compared with the results which were obtained via the approximate solution in part 1 . The approximate solution agrees extremely well with 


\begin{tabular}{|c|c|c|c|c|c|c|}
\hline$l$ & $\lambda$ & 0 & $0 \cdot 1$ & 1 & 10 & $\infty$ \\
\hline $10 \cdot 0$ & & 0.999942 & 0.999975 & 1.00013 & 1.00028 & $1 \cdot 00031$ \\
\hline $5 \cdot 0$ & & 0.999574 & 0.999835 & 1.00102 & 1.00224 & $1 \cdot 00251$ \\
\hline 3.0 & & 0.998289 & 0.999466 & 1.00487 & $1 \cdot 01051$ & 1.01180 \\
\hline $2 \cdot 0$ & & 0.995768 & 0.999598 & 1.01758 & 1.03714 & $1 \cdot 04178$ \\
\hline $1 \cdot 8$ & & 0.995174 & 1.00036 & 1.02495 & 1.05230 & 1.05892 \\
\hline $1 \cdot 6$ & & 0.995321 & 1.00256 & 1.03751 & 1.07781 & 1.08785 \\
\hline $1 \cdot 4$ & & 0.998923 & $1 \cdot 00942$ & $1 \cdot 06174$ & $1 \cdot 12603$ & $1 \cdot 14295$ \\
\hline $1 \cdot 2$ & & $1 \cdot 02046$ & $1 \cdot 03643$ & $1 \cdot 12127$ & $1 \cdot 24091$ & $1 \cdot 27664$ \\
\hline $1 \cdot 1$ & & $1 \cdot 06555$ & 1.08590 & $1 \cdot 20075$ & $1 \cdot 38898$ & $1 \cdot 45485$ \\
\hline
\end{tabular}

TABle 5. Torque ratio for a rotating sphere when the axis of rotation is parallel to a plane interface.

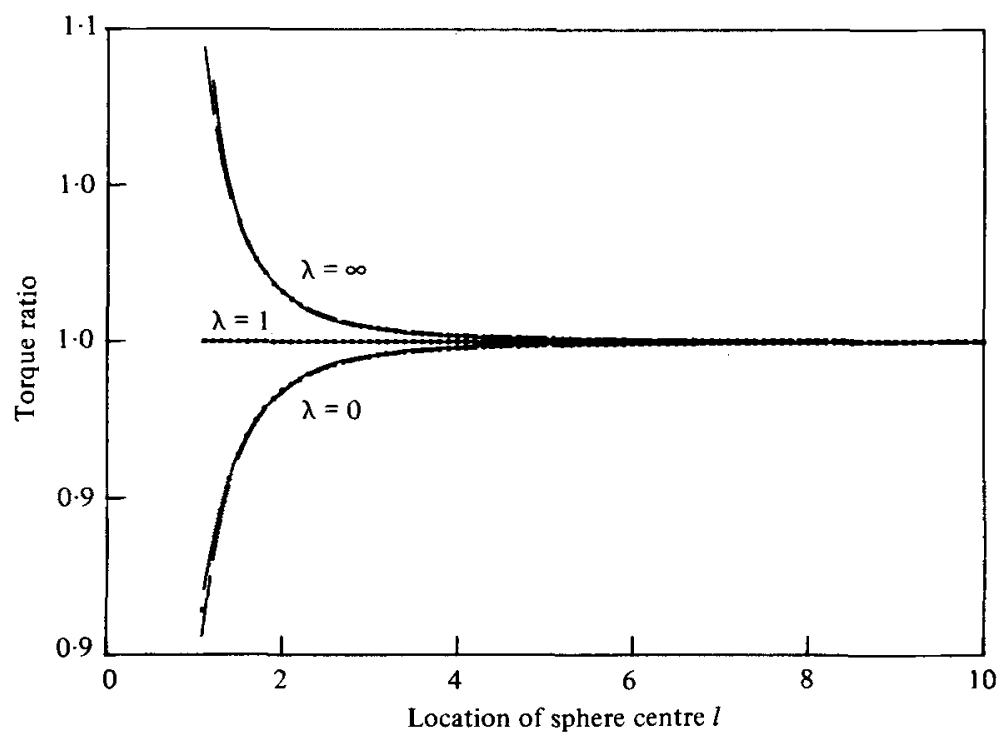

Fraure 6. Torque ratio for the rotation of a sphere when the axis of rotation is perpendicular to a plane interface. - - exact solution; - - , approximate solution; $\square \square \square$, summed series.

the exact solution for the whole range of $l$ down to $l \sim 1 \cdot 1$. Apparently, the higherorder terms in the approximate solution are insignificant in so far as the torque on the sphere is concerned.

Finally, we consider the results for rotation when the axis of rotation is parallel to the interface. The hydrodynamic torque ratio for this case is given in table 5 . According to the asymptotic theory from part 1, which was carried out to terms of $O\left(l^{-3}\right)$, the torque ratio should exceed one when $\lambda>\frac{1}{5}$ and fall below one for $\lambda<\frac{1}{5}$. This result is, of course, based on only the first term of the asymptotic expansion which is only valid for large $l$. Indeed, when the torque ratio was evaluated numerically using the exact solution of this paper, it was found to be 1.00000 when $l=10$ and $\lambda=\frac{1}{5}$. As the particle is placed closer to the interface, however, the exact solution shows that the torque ratio eventually exceeds unity even for $\lambda=0$, although the critical value of $l$ where this occurs is seen to decrease as $\lambda$ is decreased (i.e. to occur when the sphere is closer to the interface). In figure 7 , the numerically evaluated 


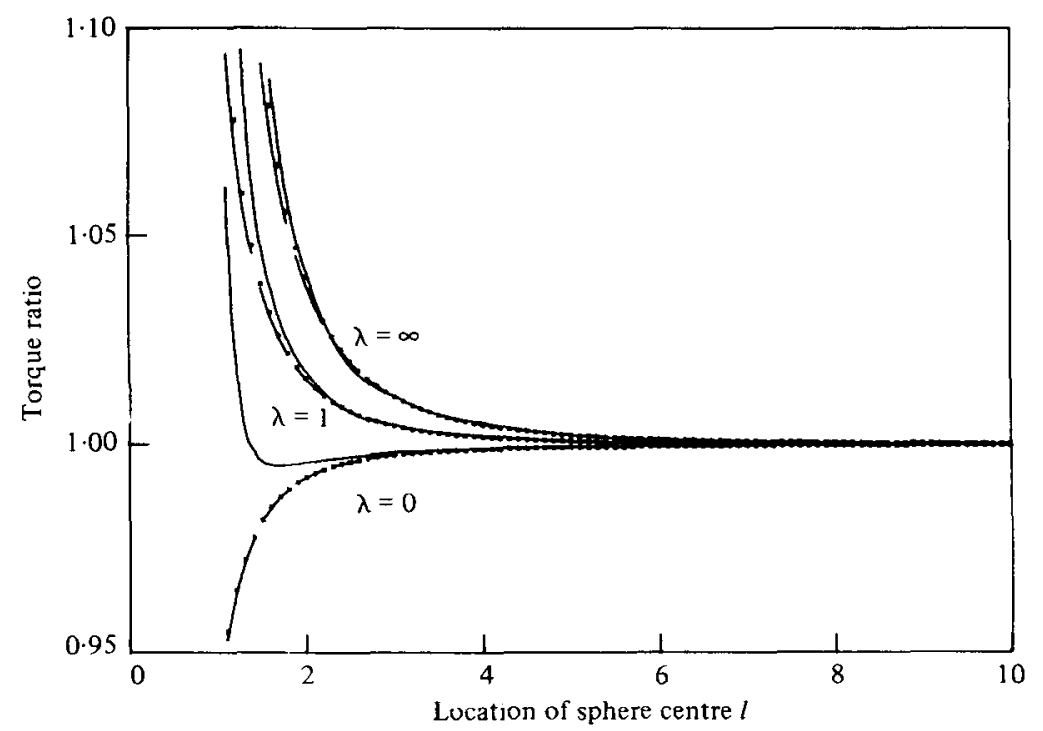

Figure 7. Torque ratio for the rotation of a sphere when the axis of rotation is parallel to a plane interface. — , exact solution; - - , approximate solution; $\mathbf{\square} \mathbf{\mathbf { a }}$, summed series.

torque ratio from this study is plotted together with the approximate (asymptotic) solution of part 1 . For $l \gg 1$, the two solutions are in excellent agreement. However, at $l \sim 2$, the two solutions diverge, especially for the case $\lambda=0$ where the asymptotic result continues to decrease below unity while the exact solution first decreases for large $l$, but then increases sharply as $l \rightarrow 1$. As before, this latter behaviour might be found in higher-order terms of the asymptotic solution, though there is no guarantee that it will appear since the expansion is only valid for $l \gg 1$. There is also a non-zero drag force generated in this problem, which is equal in magnitude but opposite in direction to the force that would have to be applied to the sphere to keep it from translating parallel to the interface (cf. Brenner 1964). However, the details of this force need not be reported here as it can be calculated directly from the torque which acts on a sphere that is translating parallel to the interface (table 3 ). Indeed, we have noted in equations (82) and (83) of part 1 that the coupling tensor which relates the force on a rotating sphere to its angular velocity should be anti-symmetric and the transpose of the coupling tensor which relates the torque on a translating sphere to its translational velocity. This reciprocal relationship between the translational and rotational problems was satisfied by the numerical results for the two problems in our present study. It should be mentioned here that the calculation of Dean \& O'Neill (1963) was numerically erroneous (cf. Goldman, Cox \& Brenner 1967) and, thus, does not agree with our results for $\lambda=\infty$.

The fact that exact results are available for the force and/or torque provides an opportunity to see whether the asymptotic results of part 1 can be improved at all. Specifically, the force and torque were found in part 1 to be related solely to the Stokeslet and rotlet strengths, and these appear to be geometric series to the level of approximation which was analysed in part 1. Since these geometric series are easily summed, it is possible that improved results for the force and torque could be obtained for $l \sim O(1)$. Obviously; there is no guarantee that additional contributions may not 


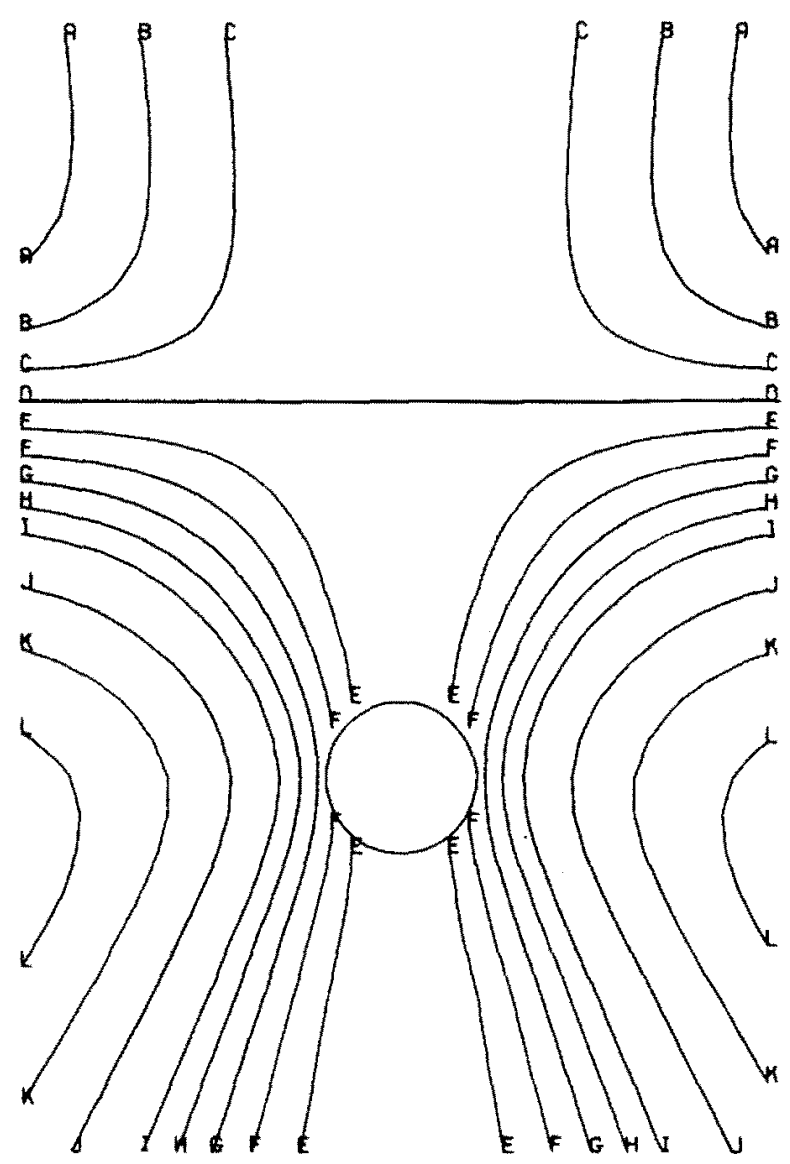

Fiaure 8. Stream function $\left[U_{c} L_{c}^{z}\right]$ for the translation of a sphere perpendicular to a plane interface $\left(\lambda=0 \cdot 1, l=5\right.$ and $\left.u_{s}=i_{k}\right) \cdot A=0 \cdot 6, B=0 \cdot 4, C=0 \cdot 2, D=0, E=-0 \cdot 2, F=-0 \cdot 4$, $\mathrm{G}=-0 \cdot 6, \mathrm{H}=-0.8, \mathrm{I}=-1 \cdot 0, \mathrm{~J}=-1 \cdot 4, \mathrm{~K}=-1 \cdot 8, \mathrm{~L}=-2 \cdot 2$.

occur at higher orders in $l^{-1}$ which would invalidate the simple geometric form which is assumed for the series in this summing process. However, we believe that it is of interest to investigate the comparison between the exact results obtained above, and the 'summed' approximate results from part 1. For the four fundamental motions of a sphere, we have compared this newly suggested approximate solution, as well as the original solution of part 1 with the exact solutions in figures $2,3,6$ and 7 . There is no difference in the numerical accuracy of the two approximate results in the region of $l \gg 1$. However, for $l \sim 1$, the 'summed' series shows somewhat improved comparison with the exact solution for most cases relative to the original solution of part 1 , though the results are still not quantitatively accurate. When the sphere is translating normal to the interface, for $\lambda=1,10$ and $\infty$, the 'summed' series reveals a more rapid increase in the drag force than occurs in the exact solution as $l \rightarrow 1$. Since the summed series is of the form $(1-\alpha / l)^{-1}$, there is a singularity indicated for $l=\alpha$ rather than $l=1$, as expected. This is, of course, both a consequence and an indication of the existence of higher-order terms in the exact solution which do not fit the geometric form that is suggested by the first few terms of the asymptotic series. The 


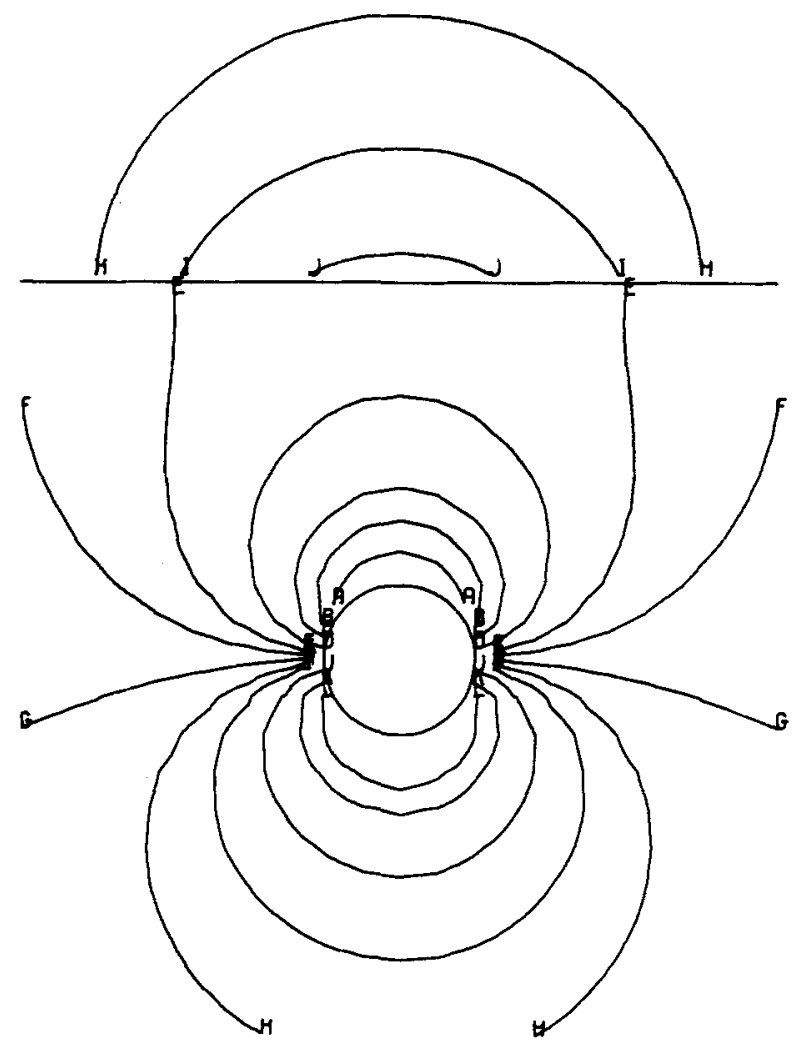

Figure 9. Pressure $\left[\mu U_{c} / L_{c}\right.$ for $z<0, \hat{\mu} U_{c} / L_{c}$ for $\left.z>0\right]$ for the translation of a sphere perpendicular to a plane interface $\left(\lambda=0 \cdot 1, l=5\right.$ and $\left.\mathbf{u}_{s}=\mathbf{i}_{z}\right) . \mathrm{A}=1 \cdot 0, \mathrm{~B}=0 \cdot 6, \mathrm{C}=0 \cdot 4$, $\mathrm{D}=0.2, \mathrm{E}=0 \cdot 1, \mathrm{~F}=0.05, \mathrm{G}=0, \mathrm{H}=-0.05, \mathrm{I}=-0 \cdot 1, \mathrm{~J}=-0.2, \mathrm{~K}=-0.4, \mathrm{~L}=-0.6$.

case $\lambda=0$ of parallel rotation, which is poorly predicted by the original asymptotic series, is not significantly improved for the same reason. The same limit, $\lambda=0$, for perpendicular translation, on the other hand, shows excellent agreement with the exact solution, suggesting the absence of higher-order terms of a fundamentally different nature for this particular limiting case.

Finally, in order to achieve a more complete understanding of the motion of a sphere in the presence of a plane interface, we have plotted pressure, velocity and vorticity fields for the translational motion of a sphere with $\lambda=0.1$ and $l=5$. When the sphere is moving normal to the interface, the flow field is axisymmetric. Thus, we show the stream function in figure 8 . The streamlines are obviously deflected owing to the presence of the 'impermeable' plane interface. The pressure field for this problem is illustrated in figure 9 . Although the dynamic pressure is positive above the sphere and negative below the sphere as would also be true in an unbounded fluid, the plane interface clearly disrupts the fore-aft anti-symmetry of the pressure field about $z=-l$. The dynamic pressure in the upper fluid is negative, and there is a pressure jump across the interface due in part to the fact that it is specified as flat. In figures 10 and 11 , the velocity components, $u$ and $w$, are plotted, respectively. In addition, the vorticity, $\omega_{\phi}$, is plotted in figure 12 . In these figures, it can be clearly seen that the presence of the impermeable interface suppresses the velocity component in the 


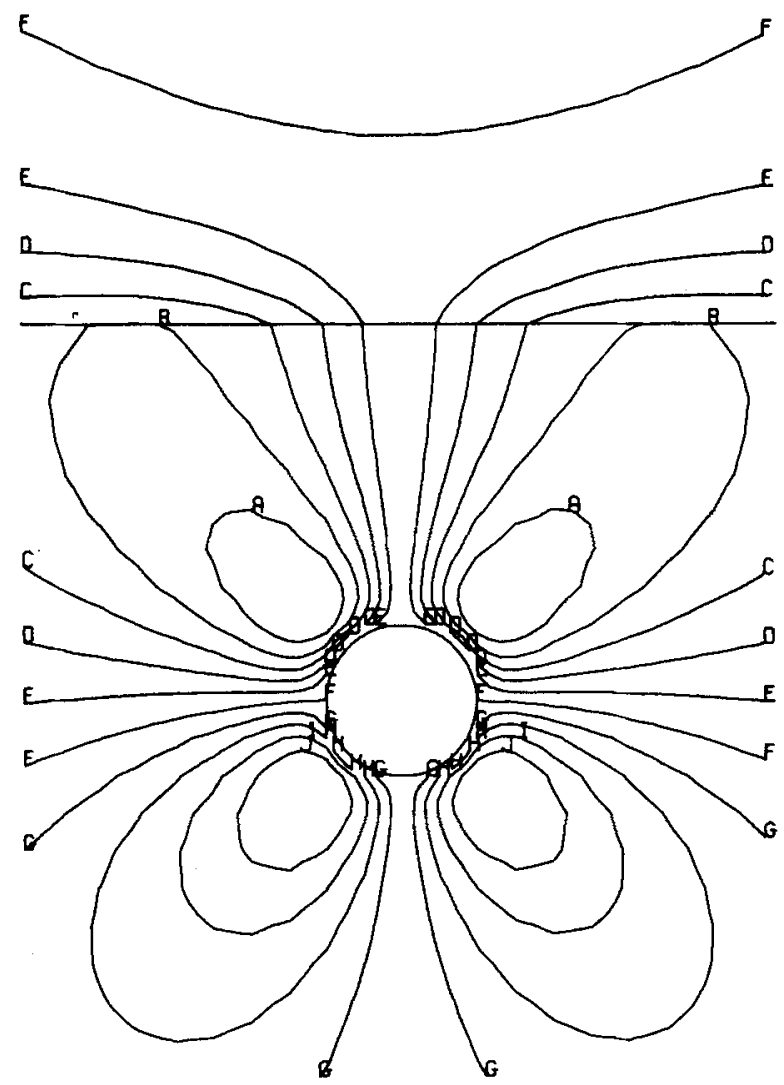

Fraure 10. $u_{r}\left[U_{e}\right]$ for the translation of a sphere perpendicular to a plane interface $(\lambda=0 \cdot 1$, $l=5$ and $\mathrm{u}_{s}=\mathrm{i}_{z}$ ). $\mathrm{A}=0 \cdot 15, \mathrm{~B}=0.12, \mathrm{C}=0.09, \mathrm{D}=0.06, \mathrm{E}=0.03, \mathrm{~F}=0, \mathrm{G}=-0.03$, $H=-0.06, I=-0.09, J=-0.12$.

$z$ direction while it enhances the velocity component in the $r$ direction owing to the small viscosity of the upper fluid. In addition, it may be noted that $\partial w / \partial r=0$ and $\partial u / \partial z<0$ at the interface. Thus negative vorticity $\omega_{\phi}$ is generated at the interface. However, as expected, strong positive vorticity $\omega_{\phi}$ is generated at the sphere surface. Hence, in the lower fluid, there exists only a small region of negative vorticity $\omega_{\phi}$ near the interface. Since the shear stress is continuous across the interface with $\lambda \neq 1$, there naturally occurs a vorticity jump across the interface.

We have also examined the flow field generated by a sphere which is translating parallel to a plane interface. We consider that the sphere is moving in $x$ direction. The pressure field is shown in figure 13. It is noted that in the lower fluid, positive dynamic pressure builds up in front of the sphere while negative dynamic pressure is found behind the sphere. However, in the upper fluid, the pressure is negative for $x>0$ and positive for $x<0$. In figures 14 and $15, u_{x}$ on $y=0$ and $u_{x}$ on $x=0$ are respectively plotted. These figures ostensibly show that the velocity gradient in the upper side of the sphere is smaller than that in the lower side of the sphere for this case of $\lambda=0 \cdot 1$. The velocity component $w$ on $y=0$ is depicted in figure 16. Although $w$ is still anti-symmetric relative to $x=0$, the anti-symmetry of $w$ relative to $z=-l$ is disrupted due to the presence of the interface. Finally, $\omega_{y}$ is plotted in figure 17. 

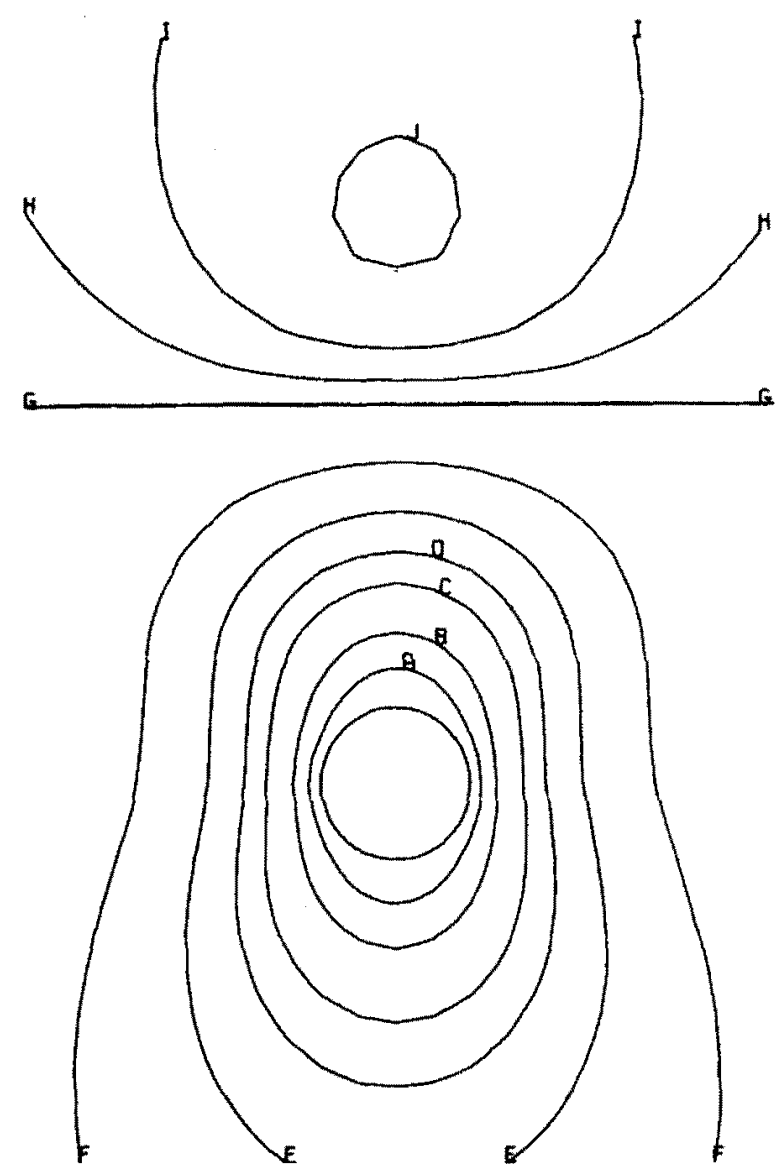

Frouke 11. $u_{x}\left[C_{0}\right]$ of the translation of a sphere perpendicular to a plane interface $\left(\lambda=0.1, l=5\right.$ and $\left.\mathbf{u}_{s}=i_{z}\right) . A=0.8, \quad B=0.6$, $\mathrm{C}=0.4, \mathrm{D}=0.3, \mathrm{E}=0.2, \mathrm{~F}=0.1, \mathrm{G}=0, \mathrm{H}=-0.03, \mathrm{I}=-0.06$, $\mathbf{J}=-0.09$.

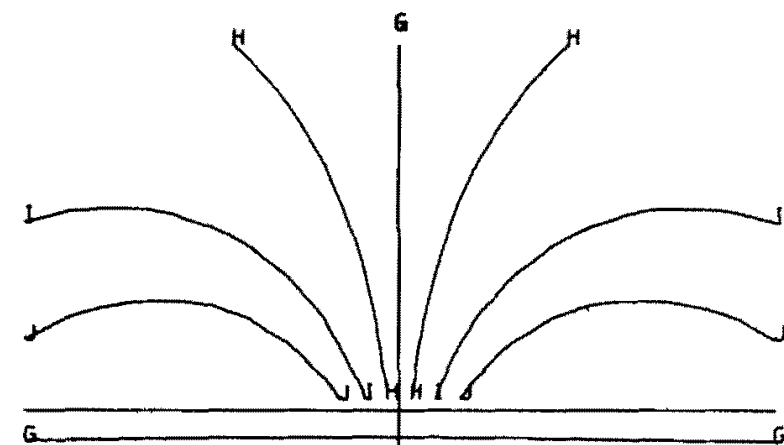

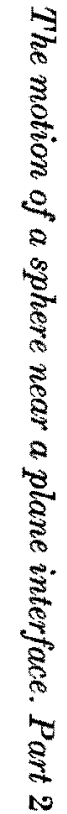

Fravae 12. $\omega_{\phi}\left[L_{c} / L_{c}\right]$ for the translation of aphere perpendicular to a plane interface $\left(\lambda=0.1 \quad l=5\right.$ and $\left.\mathrm{u}_{s}=i_{2}\right) . \mathrm{A}=0.8, \mathrm{~B}=0.4$, $\mathrm{C}=0.2, \quad \mathrm{D}=0.1, \quad \mathrm{E}=0.05, \quad \mathrm{~F}=0.025, \quad \mathrm{G}=0, \quad \mathrm{H}=-0.01$ $I=-0.03, J=-0.05$ 


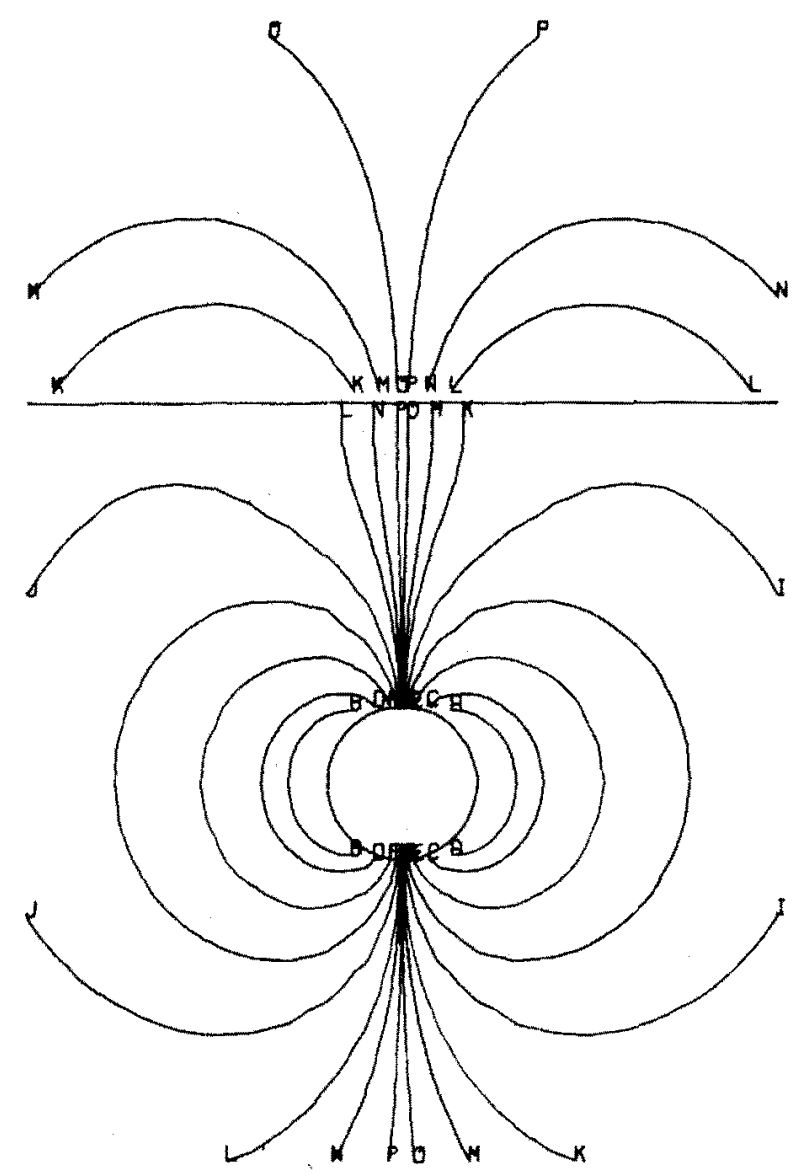

Frguke 13. Pressure $\left[\mu U_{c} / L_{c}\right.$ for $z<0, \hat{\mu} U_{c} / L_{c}$ for $\left.z<0\right]$ on $y=0$ for the translation of a sphere parallel to a plane interface $(\lambda=0 \cdot 1$, $l=5$ and $\left.\mathbf{u}_{s}=1 \mathrm{l}\right) \mathrm{A}=3 \cdot 0, \mathrm{~B}=-3 \cdot 0, \mathrm{C}=2 \cdot 0, \mathrm{D}=-2 \cdot 0, \mathrm{E}=1 \cdot 0$, $\mathrm{F}=-1 \cdot 0, \mathrm{G}=0.5, \mathrm{H}=-0.5, \mathrm{I}=0.25, \mathrm{~J}=-0.25, \mathrm{~K}=0.1$, $\mathrm{L}=-0.1, \mathrm{M}=0.05, \mathrm{~N}=-0.05, \mathrm{O}=0.01, \mathrm{P}=-0.01$.
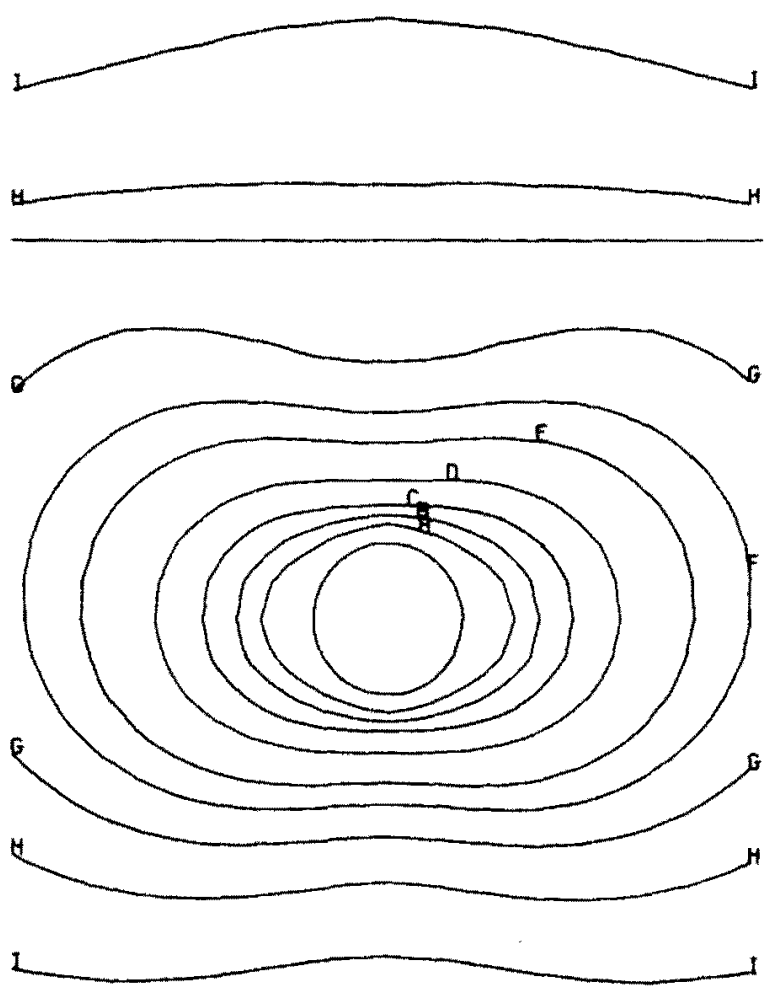

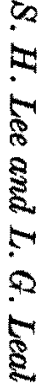

Figure 14. $u_{x}\left[U_{f}\right]$ on $y=0$ for the translation of a sphere parallel to a plane interface $\left(\lambda=0.1, l=5\right.$ and $\left.\mathbf{u}_{2}=\mathbf{i}_{2}\right) \cdot \mathrm{A}=0.8, \mathrm{~B}=0.7$ $\mathrm{C}=0 \cdot 6, \mathrm{D}=0 \cdot 5, \mathrm{E}=0.4, \mathrm{~F}=0.35, \mathrm{G}=0.3, \mathrm{H}=0.25, \mathrm{I}=0.20$. 


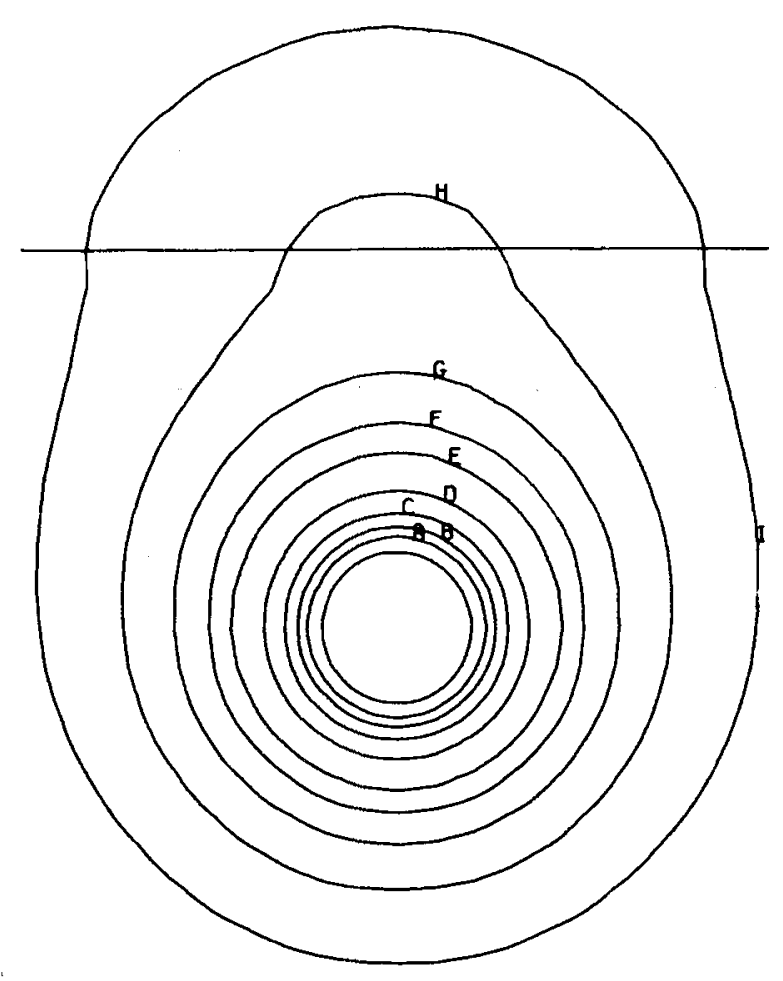

FraURe 15. $u_{x}\left[U_{t}\right]$ on $x=0$ for the translation of a sphere parallel to a plane interface $\left(\lambda=0 \cdot 1, l=5\right.$ and $\left.\mathbf{u}_{s}=\mathbf{i}_{x}\right) . \mathrm{A}=0.8, \mathrm{~B}=0.7$, $\mathrm{C}=0 \cdot 6, \mathrm{D}=0 \cdot 5, \mathrm{E}=0 \cdot 4, \mathrm{~F}=0 \cdot 35, \mathrm{G}=0 \cdot 3, \mathrm{H}=0 \cdot 25, \mathrm{I}=0 \cdot 2$.

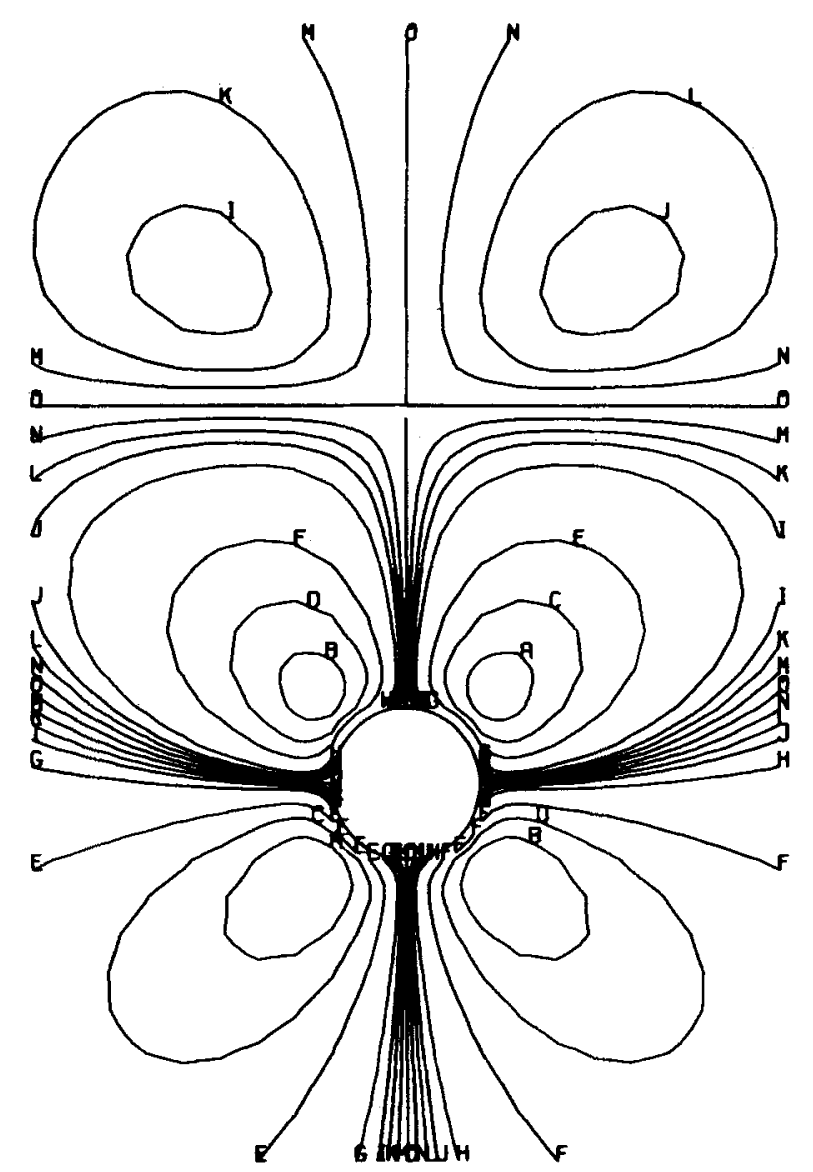

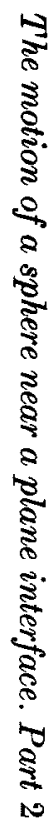

FIGURE 16. $u_{f}\left[U_{c}\right]$ on $y=0$ for the translation of a sphere parallel to a plane interface $\left(\lambda=0 \cdot 1, l=5\right.$ and $\left.\mathbf{u}_{s}=\mathbf{i}_{x}\right) \cdot A=0 \cdot 11, \mathrm{~B}=-0 \cdot 11$ $\mathrm{C}=0.08, \mathrm{D}=-0.08, \mathrm{E}=0.05, \mathrm{~F}=-0.05, \mathrm{G}=0.02, \mathrm{H}=-0.02$, $\mathrm{I}=0.012, \quad \mathrm{~J}=-0.012, \quad \mathrm{~K}=0.008, \quad \mathrm{~L}=-0.008, \quad \mathrm{M}=0.004$, $\mathrm{N}=-0.004,0=0$. 

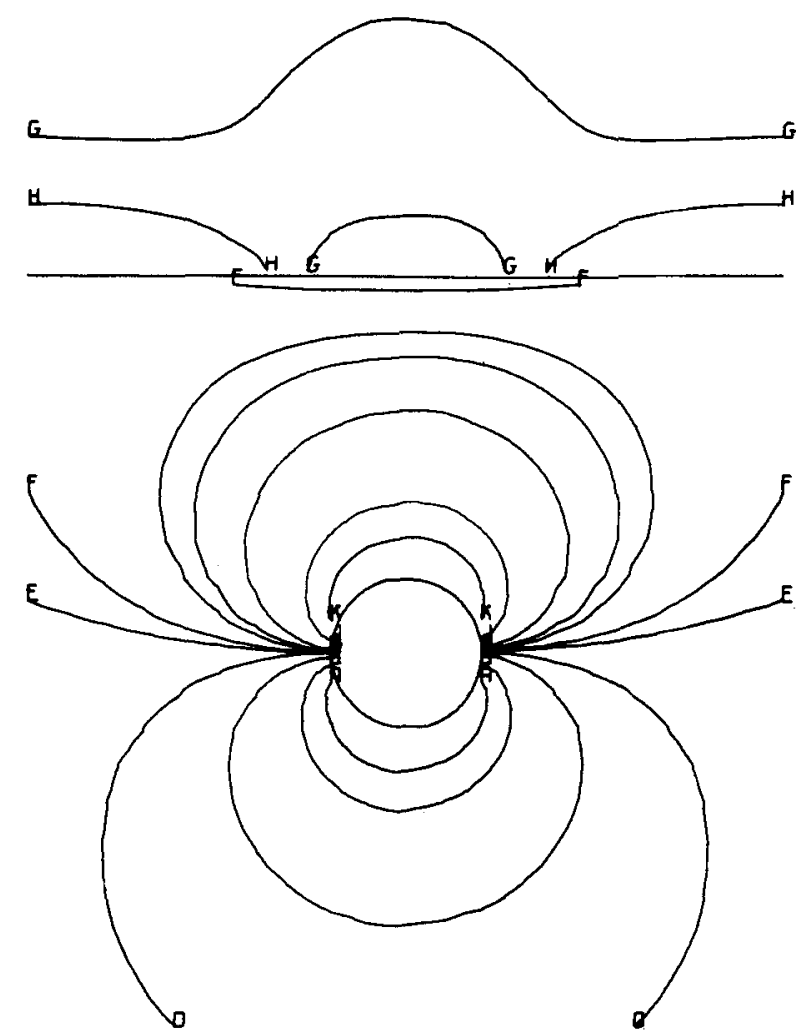

Figure 17. $\omega_{y}\left[U_{c} / L_{c}\right]$ on $y=0$ for the translation of a sphere parallel to a plane interface $\left(\lambda=0 \cdot 1, l=5\right.$ and $\left.\mathbf{u}_{s}=i_{x}\right) . \mathrm{A}=2 \cdot 0, \mathrm{~B}=1 \cdot 0, \mathrm{C}=0 \cdot 3, \mathrm{D}=0 \cdot 1, \mathrm{E}=0, \mathrm{~F}=-0 \cdot 025$, $\mathrm{G}=-0 \cdot 1, \mathrm{H}=-0 \cdot 15, \mathrm{I}=-0 \cdot 3, \mathrm{~J}=-1 \cdot 0, \mathrm{~K}=-2 \cdot 0$.

For this case, the vorticity generated by the interface has the same sign as the vorticity generated by the upper side of the sphere. The anti-symmetry of $\omega_{y}$ with respect to $z=-l$ is distorted, and there is a jump in $\omega_{y}$ across the interface as expected.

This work was supported by a grant, ENG78-10317, from the National Science Foundation.

\section{Appendix. Estimation of numerical errors in the calculated coefficients due to the truncation of terms of large $n$}

Let us express the linear equations for the system as

$$
\mathbf{a}_{n} \cdot \mathbf{x}_{n-1}+\mathbf{b}_{n} \cdot \mathbf{x}_{n}+\mathbf{c}_{n} \cdot \mathbf{x}_{n+1}=\mathbf{d}_{n} \text { for } n=1,2, \ldots
$$

We define the ratio tensor for $\mathbf{x}_{n}, \boldsymbol{\alpha}_{n}$ (a diagonal tensor), as

$$
\mathbf{x}_{n+1}=\alpha_{n} \cdot \mathbf{x}_{n}
$$

Then the equation (A 1) can be written as

$$
\mathbf{x}_{n}=\left[\mathbf{a}_{n} \cdot \boldsymbol{\alpha}_{n-1}^{-1}+\mathbf{b}_{n}+\mathbf{c}_{n} \cdot \boldsymbol{\alpha}_{n}\right]^{-1} \cdot \mathbf{d}_{n}
$$


When we truncate $\mathbf{x}_{n}$ for $n>N$, the equation (A 1 ) becomes

and

$$
\mathbf{a}_{n} \cdot \hat{\mathbf{x}}_{n-1}+\mathbf{b}_{n} \cdot \hat{\mathbf{x}}_{n}+\mathbf{c}_{n} \cdot \hat{\mathbf{x}}_{n+1}=\mathbf{d}_{n} \text { for } n=1,2, \ldots, N-1
$$

$$
\mathbf{a}_{N} \cdot \hat{\mathbf{x}}_{N-1}+\mathbf{b}_{N} \cdot \hat{\mathbf{x}}_{N}=\mathbf{d}_{N} \text { for } n=N \text {. }
$$

Here, $\hat{\mathbf{x}}_{i}$ is the calculated $\mathbf{x}_{i}$ after truncating $\mathbf{x}_{n}$ for $n>N$. Subtracting (A 1) from (A 4) and (A 5) yields

$$
\begin{array}{r}
\mathbf{a}_{N} \cdot \mathbf{E}_{n-1}+\mathbf{b}_{n} \cdot \mathbf{E}_{n}+\mathbf{c}_{n} \cdot \mathbf{E}_{n+1}=0 \text { for } n=1,2, \ldots, N-1, \quad \text { A } \\
\mathbf{a}_{N} \cdot \mathbf{E}_{N-1}+\mathbf{b}_{N} \cdot \mathbf{E}_{N}=\mathbf{c}_{N} \cdot\left[\mathbf{a}_{N+i} \boldsymbol{\alpha}_{N}^{-1}+\mathbf{b}_{N+1}+\mathbf{c}_{N+1} \cdot \boldsymbol{\alpha}_{N+1}\right]^{-1} \cdot \mathbf{d}_{N+1} \text { for } n=N,
\end{array}
$$

where $\mathbf{E}_{n} \equiv \mathbf{x}_{n}-\mathbf{x}_{n}$.

Let us define the ratio tensor for $\mathbf{E}_{n}$ as

$$
\mathbf{E}_{n+1}=\boldsymbol{\beta}_{n} \cdot \mathbf{E}_{n} .
$$

Then the error in $\hat{\mathbf{x}}_{n}$ can be simply written as

$$
\begin{aligned}
\mathbf{E}_{n}= & \hat{\mathbf{x}}_{n}-\mathbf{x}_{n}=\left\{\prod_{i=N-1}^{n}-\left[\mathbf{a}_{i} \cdot \boldsymbol{\beta}_{i-1}^{-1}+\mathbf{b}_{i}\right]^{-1} \cdot \mathbf{c}_{i} \cdot\right\}\left[\mathbf{a}_{N} \cdot \boldsymbol{\beta}_{N-1}^{-1}+\mathbf{b}_{N}\right]^{-1} \\
& \mathbf{c}_{N} \cdot\left[\mathbf{a}_{N+1} \cdot \boldsymbol{\alpha}_{N}^{-1}+\mathbf{b}_{N+1}+\mathbf{c}_{N+1} \cdot \boldsymbol{\alpha}_{N+1}\right]^{-1} \cdot \mathbf{d}_{N+1} .
\end{aligned}
$$

For simplicity, let us assume $\boldsymbol{\alpha}_{i} \sim \alpha_{i} \mathbf{I}$ and $\boldsymbol{\beta}_{i} \sim \beta_{i} \mathbf{l}$. Then, since $\left|\mathbf{a}_{i}\right|,\left|\mathbf{c}_{i}\right| \sim \frac{1}{2}\left|\mathbf{b}_{i}\right|$ for large $i$, the maximum component of $\mathbf{E}_{n}$ is

$$
\left|\mathbf{E}_{n}\right|_{\infty} \sim\left[\prod_{i=N-1}^{n} \frac{1}{\frac{1}{\beta_{i-1}}+2}\right] \frac{\left|\mathbf{d}_{N+1}\right|_{\infty}}{\left(2+\frac{1}{\beta_{N-1}}\right)\left(2+\frac{1}{\alpha_{N}}+\alpha_{N+1}\right)} .
$$

Equation (A 10) clearly shows that the truncation error in $\hat{\mathbf{x}}_{n}$ which is proportional to $\left|\mathbf{d}_{N+1}\right|_{\infty}$ becomes smaller as $n$ decreases.

\section{REFERENCES}

BART, E. 1968 The slow unsteady settling of a fluid sphere toward a flat fluid interface. Chem. Engng Sci. 23, 193.

Brenner, H. 1964 The Stokes resistance of an arbitrary particle - II. An extension. Chem. Engng Sci. 19, 599.

DeAN, W. R. \& O'NEILL, M. E. 1963 A slow motion of viscous liquid caused by the rotation of a solid sphere. Mathematika 10, 13.

FAxEN, H. 1921 Einwirkung der Gefasswände auf den Widerstand gegen die Bowegung einer kleinen Kugel in einer zähen flüssigkeit. Dissertation, Uppsala University.

Goldman, A. J., Cox, R. G. \& Brenner, H. 1967 Slow viscous motion of a sphere parallel to a plane wall. I. Motion through a quiescent fluid. Chem. Engng Sci. 22, 637.

HAppel, J. \& Brenner, H. 1973 Low Reynolds Number Hydrodynamics. Noordhoff.

JEFFERY, G. B. 1912 On a form of the solution of Laplace's equation suitable for problems relating to two sphere, Prac. Roy. Soc. A 87, 109.

JEFFERY, G. B. 1915 On the steady rotation of a solid of revolution in a viscous fluid. London Math. Soc. 14, 327.

Lee, S. H., Chadwick, R. S. \& Leal, L. G. 1979 Motion of a sphere in the presence of a plane interface. 1. An approximate solution by generalization of the method of Lorentz. $J$. Fluid Mech. 93, 705 . 
LIN, C. 1968 Statistical mechanical theory of shock waves and suspensions. Ph.D. dissertation, University of Washington.

Lorentz, H. A. 1907 A general theorem concerning the motion of a viscous fluid and a few consequences derived from it. Abhandl. Theoret. Phys. 1, 23.

O'Nert, M. E. 1964 A slow motion of viscous liquid caused by a slowly moving solid sphere. Mathematika 11, 67.

O'NeILl, M. E. \& Stewartson, K. 1967 On the slow motion of a sphere parallel to a nearby plane wall. $J$. Fluid Mech. 27, 705.

Stimson, M. \& Jeffery, G. B. 1926 The motion of two spheres in a viscous fluid. Proc. Roy. Soc. A 111, 110. 